\title{
Multiset-based assessment of vulnerability of energy infrastructures to destructive impacts
}

\author{
Igor Sheremet \\ Russian Foundation for Basic Research, Leninskiy Prosp., 32a, Moscow, Russia, \\ 119334 \\ sheremet@rfbr.ru
}

\begin{abstract}
This paper is dedicated to the application of the multigrammatical framework to the assessment of vulnerability of energy infrastructures affected by impacts destroying (reducing capabilities of) their facilities (power plants, fuel producing plants, power transmission lines, fuel transporting pipes, as well as networking devices of both electricity and fuel subsystems of an energy infrastructures). A basic graph representation of energy infrastructures is considered, and technique of their multigrammatical representation is introduced. Criterial base for recognition of the energy infrastructures vulnerability, being a generalization of the similar criterial base developed regarding industrial infrastructures is proposed. Techniques of multigrammatical modelling reservation of energy infrastructures and their recovery after impacts is proposed. Directions of future research in this area are announced.
\end{abstract}

Keywords. Energy infrastructure, vulnerability, recovery, resilience, multisets, multiset grammars, filtering unitary multiset grammars.

\section{Introduction}

The multigrammatical framework (MGF), introduced and described in [1-7], is a set of syntactically, semantically and pragmatically interconnected multiset-based knowledge representation models (KRMs) and associated with them algorithmics and implementation techniques, developed and applied to various problems from the systems analysis and operations research areas. The MGF integrates the best features of modern knowledge engineering - first of all, logic and constraint programming [8-13], providing easy and natural accumulation of knowledge bases (KBs) from atomary implications and not less easy and natural KBs' update and classical theory of optimization - namely, mathematical programming with it's refined algorithmics providing fast search of strictly optimal solutions [14-17]. The MGF, in fact, provides natural and easily modified representation of distributed sociotechnological systems (DSTSs) of different classes, as well as representation of the so called resource-based games (RBGs) being a useful and convenient tool for modelling various conflicts between DSTSs and their coalitions [18].

One of the most valuable and actual areas of the MGF application is an assessment of DSTSs' resilience/vulnerability to various destructive impacts (malfunctions, technogenic catastrophes, natural hazards, acts of terror, mutual sanctions etc.). A unified approach to the solution of this class of problems regarding largescale industrial systems (ISs) was described in $[3,4,7]$, whilst techniques of the MGF application to an assessment of resilience of modern intelligent transport systems - in [19]. However, a background of all modern DSTSs is an energy infrastructure (EI), providing production and delivery necessary amounts of electric power

Copyright (C) 2021 for this paper by its authors. Use permitted under Creative Commons License Attribution 4.0 International (CC BY 4.0). 
and fuel to various stationary and mobile consumers, including industrial facilities, living houses, transportation vehicles etc [20-22].

This paper is dedicated namely to the application of the MGF to some considered from the substantial and mathematical points of view in [22-27] actual tasks from the area of resilience of energy infrastructures. Amounts of electric power (EP) to be delivered by an EI on demand of external customers at some predefined period of time in a general case are restricted by amounts of primary resources - crude oil, natural gas, and other possible energy carriers (ECs) - available for EP generation, as well as by limited bandwidths of links forming electric grids and fuel pipelines. A problem in question is, given a demand of costumers, i.e. amounts of power and fuel to be consumed by them during a considered time period (this demand will be named also an order), an EI segment, including fuel producing and power generating facilities, links providing power transmission and fuel transfer through distributed areas, as well as terminal units delivering fuel and power to their consumers, primary resources available for power generation, a destructive impact, eliminating some part of a considered EI segment and the aforementioned resources, to assess whether a part of a considered segment and resources, remained after an impact, would be capable to produce and deliver amounts of power and fuel necessary to consumers (in other words, to complete an order). If so, then an EI will be named resilient to this impact. Otherwise an EI will be named vulnerable to it. The objective of this paper is to develop a criterial base providing the assessment of EIs vulnerability to destructive impacts. Everywhere below in this paper we shall consider an EI as a closed system, which operate without direct application of any external resources or their application for replenishment of EI own (internal) resources spent whilst order completion.

A content of this paper is as follows. A basic graph representation of EIs is introduced and discussed in the Section 2. Filtering unitary multiset grammars being a basic tool for consideration and solution of the problem in question are described in the Section 3. A multigrammatical representation of energy infrastructures is proposed in the Section 4 whilst criteria of vulnerability of energy infrastructures to destructive impacts - in the Section 5. Modelling reservation of EIs and their recovery after impacts is considered in the Section 6. A Conclusion is dedicated to the future directions of the MGF development and it's application to various issues concerning resilience of critical infrastructures and key resources.

\section{Basic graph representation of energy infrastructures}

An energy infrastructure is usually considered consisting of two strongly interconnected and mutually supplying segments producing fuel and electricity [20-22].

An electricity infrastructure (ElcI) in the most general case contains generation facilities (power plants, PPs), power transforming-distributing substations (PTDSs), and power terminal units (PTUs), delivering electric power to it's consumers. All these elements are connected by links, named power transmission lines (PTLs), each such line having it's own technical parameters (voltage, length, power losses during transmission etc.), and are joined to electric grids, which, in fact, in aggregate form ElcI [21-23].

A fuel infrastructure (FI) $[24,25,28,29]$, similarly to an ElcI, includes fuel producing plants (FPPs), working out fuel from some primary energy carriers (PECs), and fuel distribution stations (FDSs), as well as fuel terminal units (FTUs). All these elements are connected by pipes, which, in a general case, as PTLs, have individual technical parameters (diameter, length, pressure, amounts of EP consumed, fuel losses during transfer etc.). Fuel produced by FPPs is used by power plants and other consumers. To limit a complexity of consideration here, we shall not expand a FI down to production crude oil and natural gas from oil and gas fields and their transportation via oil and gas pipelines to FPPs; we shall assume that certain amounts of primary energy carriers (PECs), used for fuel production, are accumulated at fuel storages (FSs) collocated with FPPs, and these amounts are a part of a resource base (RB) of an EI.

ElcI and FI are joined with one another by terminal units: any element of an FI consumes an electric power delivered to it by some PTU, whilst any PP is operating due to a FTUs delivering fuels needed for power generation (in a general case there may be several energy carriers utilized by a single power plant). Also there are PTUs and FTUs delivering power and fuels to external consumers. Regarding a considered time period (hour, day etc.), any FPP may produce certain amounts of various fuels, as well as any PP may produce certain 
amounts of EP with various technical parameters. Any output of any element of EI is assumed consistent with a link transferring resource from it to another element, which input, in turn, is assumed consistent with the aforementioned link which is an incoming for this another element and thus delivering to it the aforementioned resource. This overlapping of EI elements and boundary points of EI links is a background for modelling a circulation of an EP and fuel via EI. Any link has a limited bandwidth (or throughput capacity) as an integral technical parameter, determining maximal amount of power (if it is a PTL) or fuel (if it is a pipe) which may be transmitted (transferred) via this link during a considered time period. Also, as it was mentioned above, there are some power losses occurring during it's transmission via a PTL; similar losses of fuel are inherent to fuel transferring pipes.

So both electricity and fuel infrastructures have a tree-like concentric topology and, based on the above, an EI may be represented by an weighted oriented graph with nodes corresponding to EI elements, and marked edges corresponding to EI links. This graph, in turn, in the algebraic representation is a ternary relation $G \subseteq A \times A \times \boldsymbol{N}$, where $A$ is a set of EI elements (PPs, PTDSs, PTUs, FPPs, FDSs, FTUs, FSs), and $\boldsymbol{N}$ is a set of positive rational numbers representing bandwidths of EI links (PTLs and pipes). So $\left\langle a, a^{\prime}, n\right\rangle \in G$ means that an element $a$ is capable to transmit (transfer) to an element $a^{\prime}$ amount of resource (EP or fuel) by link (PTL or pipe) $\left\langle a, a^{\prime}\right\rangle$ no more than $n$ units (kilowatt·hours in the case of EP, and barrels, cubic meters, kilograms, tons etc. in the case of various fuels) during a considered time period. There may be the only triple $<a, a^{\prime}, n>\in G$ for any link $\left\langle a, a^{\prime}>\right.$, i.e. a link has the only bandwidth (throughput capacity).

A destructive impact, which in a general case is distributed, may eliminate some elements or/and links of an EI as well as some amounts of resources stored at an EI resource base; naturally, an impact may be represented by some subset of nodes and edges eliminated from an initial graph $G$.

Let us illustrate the said by an example.

Example 1. Consider a small hypothetical segment of some EI including a power plant, two power transformation-distribution stations, seven power terminal units, a fuel producing plant, a fuel storage, two fuel distribution stations, and three fuel terminal units (figure 1(a)). (Sequential numbers of FDSs and FTUs, as well as names of fuel storage and fuel producing plant are denoted by bold symbols).

There are also three external power customers. Generated power from a PP is delivered to both PTDSs, the first of which (enumerated "1") delivers received power to four PTUs ( "1", "2", "3" and "7"), and the second ("2") delivers accepted power to five PTUs ("4", "5", "6", "8" and "9"). PTUs deliver power to the following elements of the EI: PTU “1” - to FDS “2”, PTU “2” - to FDS “1”, PTU “3” - to FPP, PTU “4” - to FS, PTU " 5 " - to the power customer " 1 ", "6" - to the power customer " 2 ", "7" - to the power customer " 3 ". In turn, elements of FI by consumption of electric power deliver fuel as follows: FS - to FPP, FPP - to FDS “1”, FDS “1” - to FDS “2”, FDS “2” - to FTU “1” collocated with power plant. FTUs "2" and "3", which both receive fuel from FDS “1", deliver fuel to fuel customers "1" and "2" respectively. These FTUs are provided by electric power from PTUs "8" and "9", receiving power from the PTDS "2". An algebraic representation of the considered graph, including bandwidths (throughput capacities) is contained in the table 1.

The impact destroys the PTDS "1", PTUs "5" and "7", as well as the FDS "2". Along with these destructions the impact reduces bandwidth of the link between the PTDS " 2 " and the PTU " 6 " from $300 \mathrm{kWh}$ to $100 \mathrm{kWh}$. The resulting graph of the affected EI is represented at figure 1(b).

Having this basic graph representation of EIs we may move to the MGF application to the assessment of resilience/vulnerability of EIs. To introduce proposed a criterial base for this assessment let us remind some necessary notions and denotations concerning syntax and semantics of filtering unitary multiset grammars (FUMGs) being a simplest MGF tool for formalizing and solution of many actual tasks from the applied systems analysis and operations research areas. 


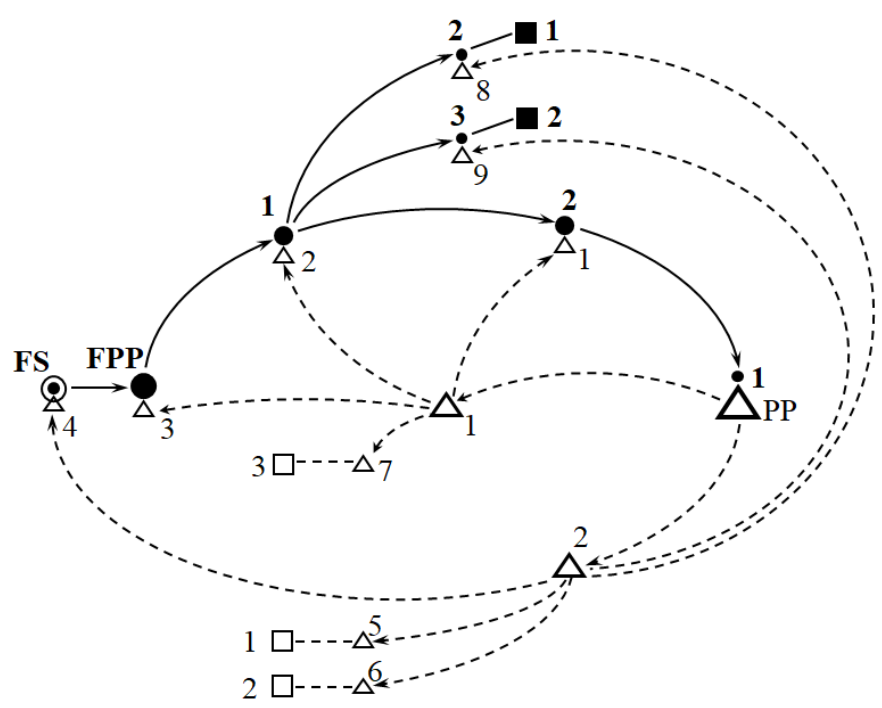

a)

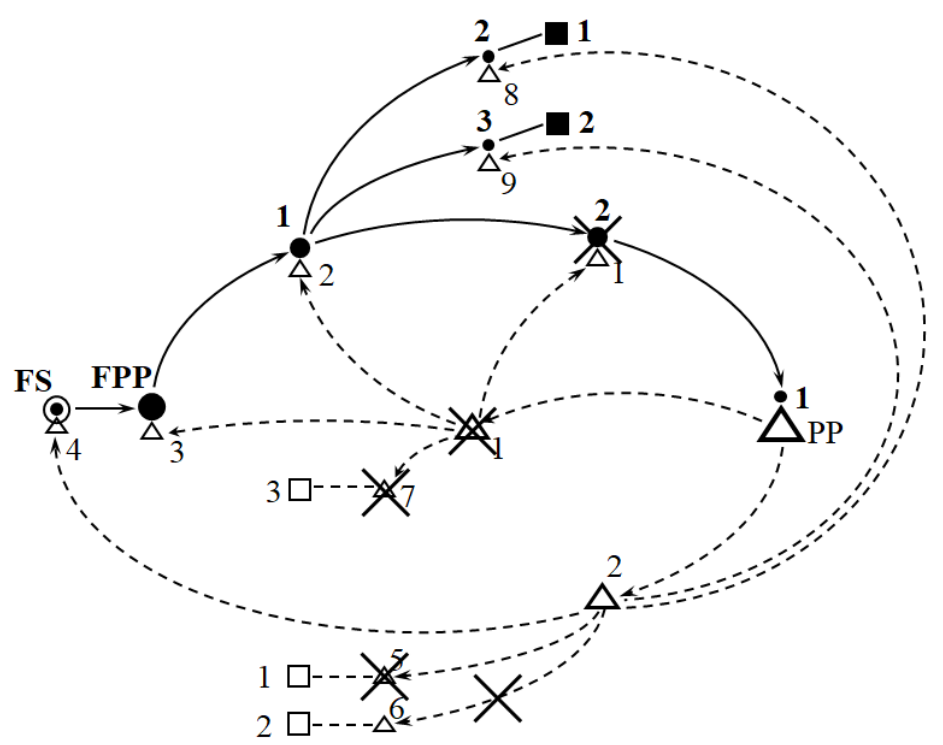
--.- - electric power

b)

Figure 1. Graph representation of a segment of an energy infrastructure a) initial state, b) state after impact.

Table 1. An algebraic representation of the graph

\begin{tabular}{|l|l|l|l|}
\hline № & Source point & Receiver point & $\begin{array}{c}\text { Channel upper threshold } \\
\text { values of bandwidths } \\
\text { (throughput capacities) }\end{array}$ \\
\hline 1 & PP & PTDS1 & $1000 \mathrm{kWh}$ \\
\hline 2 & PP & PTDS2 & $1100 \mathrm{kWh}$ \\
\hline 3 & PTDS1 & PTU1 & $200 \mathrm{kWh}$ \\
\hline 4 & PTDS1 & PTU2 & $300 \mathrm{kWh}$ \\
\hline 5 & PTDS1 & PTU3 & $400 \mathrm{kWh}$ \\
\hline 6 & PTDS1 & PTU7 & $100 \mathrm{kWh}$ \\
\hline 7 & PTDS2 & PTU4 & $200 \mathrm{kWh}$ \\
\hline 8 & PTDS2 & PTU5 & $300 \mathrm{kWh}$ \\
\hline 9 & PTDS2 & PTU6 & $300 \mathrm{kWh}$ \\
\hline
\end{tabular}




\begin{tabular}{|l|l|l|l|}
\hline № & Source point & Receiver point & $\begin{array}{c}\text { Channel upper threshold } \\
\text { values of bandwidths } \\
\text { (throughput capacities) }\end{array}$ \\
\hline 10 & PTDS2 & PTU8 & $200 \mathrm{kWh}$ \\
\hline 11 & PTDS2 & PTU9 & $100 \mathrm{kWh}$ \\
\hline 12 & PTU5 & EPC1 & $300 \mathrm{kWh}$ \\
\hline 13 & PTU6 & EPC2 & $300 \mathrm{kWh}$ \\
\hline 14 & PTU7 & EPC3 & $100 \mathrm{kWh}$ \\
\hline 15 & FS & FPP & 200 tons of crude oil \\
\hline 16 & FPP & FDS1 & 200 tons of the fuel \\
\hline 17 & FDS1 & FDS2 & 100 tons of the fuel \\
\hline 18 & FDS1 & FTU2 & 50 tons of the fuel \\
\hline 19 & FDS1 & FTU3 & 50 tons of the fuel \\
\hline 20 & FDS2 & FTU1 & 100 tons of the fuel \\
\hline 21 & FTU1 & PP & 100 tons of the fuel \\
\hline 22 & FTU2 & FC1 & 50 tons of the fuel \\
\hline 23 & FTU3 & FC2 & 50 tons of the fuel \\
\hline
\end{tabular}

\section{Filtering unitary multiset grammars}

Following [1, 2, 3], we shall define a multiset grammar (multigrammar, MG) as a couple

$$
S=<v_{0}, R>\text {, }
$$

where a multiset (MS)

$$
v_{0}=\left\{n_{1} \cdot a_{1}, \ldots, n_{m} \cdot a_{m}\right\}
$$

is called a kernel, and $R$, called a scheme, is a finite set of rules which are applied for generation new multisets from already generated. (Everywhere below objects are denoted $a, a_{i}, a_{i_{j}}$, whilst their multiplicities being positive rational numbers - as $n_{i}, m_{i}, n_{i_{j}}$ etc.; a construction $n_{i} \cdot a_{i}$ representing collection of $n_{i}$ objects $a_{i}$ is called a multiobject). A rule has a form

$$
v \rightarrow v^{\prime}
$$

where $v$ and $v^{\prime}$, called respectively the left part and the right part of a rule, are multisets, and $v \neq\{\varnothing\}$. By $A_{s}$ we shall designate below a set of all objects having place in rules entering a scheme $R$ of an MG $S$.

The semantics of a rule is defined on the background of the relation of inclusion on multisets, denoted $\subseteq$, and operations of addition and subtraction of multisets, denoted respectively $\boldsymbol{+}$ and $\boldsymbol{-}$. Let $\bar{v}$ be a multiset. A rule (3) is applicable to $\bar{v}$, if

$$
\bar{v} \subseteq v
$$

and a result of an application is a multiset

$$
\bar{v}^{\prime}=\bar{v}-v+v^{\prime}
$$

i.e. if $\bar{v}$ includes $v$, then $v$ is replaced by $v^{\prime}$. This operation is called a generation step, providing a generation an MS $\bar{v}^{\prime}$ from an MS $\bar{v}$ by application a rule $r \in R$, that is denoted as

$$
\bar{v} \stackrel{r}{\Rightarrow} \bar{v}^{\prime}
$$


whilst a fact, that an MS $\bar{v}^{\prime}$ is generated from an MS $\bar{v}$ by any (including empty) sequence of generation steps, called a generation chain, is recorded as

$$
\bar{v}_{\Rightarrow}^{R} \bar{v}^{\prime}
$$

or, if the only MG is considered, then, as in the classic string-operating grammars [28, 29],

$$
\bar{v}_{\Rightarrow}^{*} \bar{v}^{\prime}
$$

If a generation chain is non-empty, a denotation $\stackrel{+}{\Rightarrow}$ instead of $\stackrel{*}{\Rightarrow}$ is used.

A set of multisets (SMS), generated by an MG $S=\left\langle v_{0}, R\right\rangle$, is denoted $V_{S}$ and is defined as follows:

$$
V_{s}=\left\{v \mid v_{0} \stackrel{R}{\Rightarrow} v\right\}
$$

An MS $v$ is called a terminal multiset (TMS), if there is no one rule $r \in R$ which may be applied to $v$. A set of terminal sets (STMS) will be denoted $\bar{V}_{s}$. Obviously, $\bar{V}_{s} \subseteq V_{s}$.

Unitary multiset grammars (UMGs) are a simplified version of a partial case of MGs, called context-free multigrammars. A scheme of an UMG is a set of unitary rules (URs), where an UR is recorded as

$$
a_{i_{0}} \rightarrow m_{1} \cdot a_{i_{1}}, \ldots, m_{k} \cdot a_{i_{k}}
$$

that is equivalent to

$$
\left\{1 \cdot a_{i_{0}}\right\} \rightarrow\left\{m_{1} \cdot a_{i_{1}}, \ldots, m_{k} \cdot a_{i_{k}}\right\}
$$

The left part of an UR being an object $a_{i_{0}}$ is called it's header, whilst the right one - it's body. A set of nonterminal objects, each being a header of at least one UR, is denoted $A_{S}^{N}$; and a set of all other objects, presenting only in bodies of URs and called terminal, is denoted $\overline{A_{S}}$ :

$$
\begin{gathered}
A_{S}=A_{S}^{N} \cup \overline{A_{S}}, \\
A_{S}^{N} \cap \overline{A_{S}}=\{\varnothing\}, \\
A_{S} \subseteq \boldsymbol{V}^{+},
\end{gathered}
$$

where $\boldsymbol{V}^{+}$is a set of non-empty strings in some primary alphabet $\boldsymbol{V}$ used for construction of objects' names. Everywhere below bold letters in objects' names will be assumed entering an alphabet $\boldsymbol{V}$, and bold letters "(“", ")", "[", "]", ":" will be delimiters entering $\boldsymbol{V}$ and used for construction of object names entering a set $A_{S}$.

UMGs may be classified by number of URs having the same header. If an UMG $S=<v_{0}, R>$ is such that in a scheme $R$ there exists at least one non-terminal object being of header of $m>1$ URs, then this UMG is named alternating; otherwise, i.e. if any non-terminal object is a header of the only one UR, then this UMG is named non-alternating. Evidently, if an UMG $S$ is non-alternating, then it defines a one-element STMS, i.e. $\left|\bar{V}_{S}\right|=1$. If an UMG $S$ is alternating, then in a general case it defines a set containing no less than one TMS, i.e. $\left|\bar{V}_{S}\right| \geq 1$.

Also UMGs may be cyclic or non-cyclic. An UMG $S=\left\langle v_{0}, R\right\rangle$ will be called cyclic, if there exists a generation chain $v_{0} \stackrel{*}{\Rightarrow} v \stackrel{+}{\Rightarrow} v^{\prime}$ such that $v \subseteq v^{\prime}$, or, just the same, $v^{\prime}=v+\Delta v$, where $\Delta v \supseteq\{\varnothing\}$. As may be seen, a cyclic UMG in a general case, when $\Delta v \supseteq\{\varnothing\}$ but $\Delta v \neq\{\varnothing\}$, defines an infinite STMS $\bar{V}_{s}$. All UMGs which are not cyclic, are named acyclic. Any acyclic UMG $S=\left\langle v_{0}, R\right\rangle$ defines a finite STMS $\bar{V}_{s}$. 
By finite or infinite number of elements of an STMS defined by an UMG $S=\left\langle v_{0}, R\right\rangle$ it may finitary (in this case $\left|\bar{V}_{S}\right|<\infty$ ) or infinitary (in this case $\left|\bar{V}_{S}\right|=\infty$ ). As it is known from [1,2], any infinitary UMG is obligatory cyclic, while any finitary UMG is acyclic. There exist cyclic UMGs being finitary.

Alternating UMGs are a standard tool for representation of alternative structures of complex (composite) objects or ways of solution of some task. This class of UMGs is for a long time used for modelling industrial systems and infrastructures [1-7]. From the other side, cyclic UMGs may be applied to a description of interconnected processes and critical infrastructures with mutual resource exchange; for example, a fuel infrastructure produces a fuel which is consumed by an electricity infrastructure, in turn, providing operation of facilities of a FI. Such UMGs will be applied below in this paper for representation and consideration of energy infrastructures.

Example 2. Consider the UMG $S=\left\langle v_{0}, R>\right.$, where $v_{0}=\{2 \cdot($ auto) $\}$, and the scheme $R$ contains three unitary rules $r_{1}, r_{2}$ and $r_{3}$ :

$$
\begin{gathered}
r_{1}:(\text { auto }) \rightarrow \\
1 \cdot(\text { frame }), 1 \cdot(\text { engine }), 4 \cdot(\text { wheel }), 4 \cdot(\text { door }), \\
400 \cdot(\text { kWh }), 50 \cdot(\text { mnt }: \text { autos } \text { AL }) ; \\
r_{2}:(\text { engine }) \rightarrow 1 \cdot(\text { motor }), 1 \cdot(\text { fuel tank }), \\
100 \cdot(\text { kWh }), 60 \cdot(\text { mnt }: \text { engines } 1 \text { AL }) ; \\
r_{3}:(\text { engine }) \rightarrow 1 \cdot(\text { motor }), 1 \cdot(\text { fuel tank }), \\
80 \cdot(\text { kWh }), 70 \cdot(\text { mnt }: \text { engines } 2 A L) .
\end{gathered}
$$

The kernel of this UMG represents the order, which objective is to obtain two autos, whilst the scheme represents the so called manufacturing technological base of some industrial facility capable to complete such orders. The UR $r_{1}$ represents the structure of auto, which consists of frame, engine, 4 wheels and 4 doors, as well as resources necessary for assembling this auto: 400 kilowatt hours of electric power and 50 minutes of operation of autos assembling line (AL). The URs $r_{2}$ and $r_{3}$ represent structure of engine (motor and fuel tank), and two alternative ways of it's manufacturing by two engines assembling lines, the first consuming 100 kilowatt·hours and 60 minutes, and the second - 80 kilowatt-hours and 70 minutes for one engine. According to the semantics of UMGs, $\bar{V}_{s}=\left\{v_{2,0}, v_{0,2}, v_{1,1}\right\}$, where $v_{2,0}$ represents total of resources necessary for manufacturing both autos by the first way (involving the first engines $\mathrm{AL}$ ), $v_{0,2}$ - similar value when both engines are assembled by the second such AL, and $v_{1,1}-$ when engines are assembled in parallel by separate ALs. Evidently,

$$
\begin{gathered}
v_{2,0}=v+\{1000 \cdot(\boldsymbol{k W h}), 120 \cdot(\text { mnt: engines } 1 \text { AL })\} \\
v_{0,2}=v+\{960 \cdot(\boldsymbol{k W h}), 140 \cdot(\text { mnt }: \text { engines } 2 A L)\} \\
v_{1,1}=v+\{980 \cdot(\boldsymbol{k W h}), 60 \cdot(\text { mnt: } \text { engines } 1 \text { AL }), 70 \cdot(\text { mnt: engines } 2 A L)\}
\end{gathered}
$$

where

$$
\boldsymbol{v}=\left\{\begin{array}{c}
2 \cdot(\text { frame }), 2 \cdot(\text { engine }), 8 \cdot(\text { wheel }), 8 \cdot(\text { door }), 2 \cdot(\text { motor }), \\
2 \cdot(\text { fuel tank }), 100 \cdot(\text { mnt }: \text { autos } A L)
\end{array}\right\}
$$

We shall use below filtering unitary multiset grammars (FUMGs) as a basic mathematical tool for representation and solution of tasks in question. According to [1,2], a FUMG is a triple

$$
S=<v_{0}, R, F>
$$

where an UMG $S^{\prime}=\left\langle v_{0}, R>\right.$ is called a core UMG of a FUMG $S$, and $F$ is a filter, i.e. a set of so called boundary and optimizing conditions on multiplicities of objects specified in a filter. A filter provides selection from an STMS, generated by an UMG $S^{\prime}$, terminal multisets satisfying aforementioned conditions. A boundary 
condition (BCs) is recorded as $a \theta n$ or ' , where $\theta \in\{\geq,>,<, \leq,=, \neq\}$, whilst an optimizing condition (OC) is recorded as $a=o p t$, where opt $\in\{\min , \max \}$. So in a general case

$$
F=F_{\leq} \cup F_{\text {opt }},
$$

where $F_{\leq}$is a set of BCs, and $F_{o p t}$ is a set of OCs. Semantics of filters, in fact, is very similar to semantics of relational query languages if to consider a set $\bar{V}_{s^{\prime}}$ as a specific database (however, infinite in a general case); also, due to application of OCs, filters provide natural representation of various tasks from the area of mathematical programming and, in general, operations research [1,2]. Formally, semantics of UMGs and FUMGs are interconnected by the following relation:

$$
\bar{V}_{s}=\left(\bar{V}_{s^{\prime}} \downarrow F_{\leq}\right) \downarrow F_{\text {opt }},
$$

where symbol $\downarrow$ denotes an operation of filtration: an STMS, generated by an UMG $S^{\prime}$, is filtered by a set of $\mathrm{BCs}$, and then a resulting subset, including TMSs, satisfying all BCs entering $F_{\leq}$, is filtered by a set of OCs, so, finally, $\bar{V}_{s}$ includes TMSs satisfying not only all BCs but also all OCs.

Example 3. Let us consider now the FUMG $S=\left\langle v_{0}, R, F\right\rangle$, where $v_{0}$ and $R$ are the same as above, and

$$
F=\{(\text { mnt: engines } 1 A L)>0,(\text { mnt: engines } 2 A L)>0\} .
$$

From the substantial point of view this filter provides selection of such ways of order completion where no one engines $\mathrm{AL}$ is out of operation (both such assembling lines are involved). So, obviously, $\overline{V_{s}}=\left\{v_{1,1}\right\}$. If

$$
F=\{(\boldsymbol{k} \boldsymbol{W h})=\min \}
$$

i.e. such ways of order completion are preferable which consume minimal amount of electric power, then $\bar{V}_{s}=$ $\left\{v_{0,2}\right\}$. In the case

$$
\begin{gathered}
F=\{(\boldsymbol{m n t}: \text { engines } 1 \boldsymbol{A L})>0,(\boldsymbol{m n t} \text { : engines } 2 \boldsymbol{A L})>0,(\boldsymbol{k W h})=\min \}, \\
\bar{V}_{S}=\left\{v_{1,1}\right\} \downarrow\{(\boldsymbol{k W h})=\min \}=\left\{v_{1,1}\right\} .
\end{gathered}
$$

According to features of their core UMGs, filtering UMGs may be alternating or non-alternating, cyclic and acyclic, finitary or infinitary. However, due to an application of it's filter a FUMG, which core UMG is infinitary, may be finitary [1, 2]: a filter may select a finite subset of an infinite STMS defined by a core UMG of an FUMG.

Now, at last, we may move directly to the application of FUMGs to the assessment of resilience/vulnerability of energy infrastructures, beginning from a multigrammatical representation of EIs.

\section{Basic multigrammatical representation of energy infrastructures Let us begin from an electricity infrastructure.}

We shall use in URs below names of objects which syntax will be ( $\boldsymbol{k} \boldsymbol{W} \boldsymbol{h}: p)$, where the string $\boldsymbol{k} \boldsymbol{W h}$ denotes a measurement unit of EP transmitted via PTLs (kilowatt-hour), and $p$ is a string in an alphabet $\boldsymbol{V}$ representing a geographical point, where an element of an ElcI is located (it may be designated by a unique symbolic name associated with specific geographic coordinates in a special database, or directly by these coordinates). So a multiobject $n \cdot(\boldsymbol{k W h}: p)$ represents $n$ kilowatts generated or consumed at a point (position, place) $p$.

Let us begin our consideration from power terminal units. Any PTU in order to deliver one unit of power to a consumer, switched to this PTU, must receive it from a closest PTDS, connected with it by a PTL. So a unitary rule, representing this fragment of an ElcI, would be as follows: 


$$
(\boldsymbol{k W h}: p t u) \rightarrow n \cdot(\boldsymbol{k W h}: p t d s), n \cdot[p t d s, p t u]
$$

where $p t u$ and $p t d s$ are strings, representing locations of, respectively, a PTU and a supplying it PTDS, whilst $[p t d s, p t u]$ is a string, representing a connecting them PTL. In other words, [ptds, ptu] is an object representing a PTL, which start and final points are respectively $p t d s$ and $p t u$. A value $n \geq 1$ depends, finally, on amounts of power losses occurring during it's transmission via a PTL (in the case $n=1$ there are no any such losses); $n$ is a rational number. So a multiobject $n \cdot[p t d s, p t u]$ represents a fact that a considered PTL provides transmission of one kilowatt-hour to a PTU located at a point $p t u$, receiving $n$ kilowatt-hours from a PTDS located at a point $p t d s$. (Let us note that the sense of (29) is fully similar to the sense of (10) regarding industrial systems and called a technological interpretation of unitary rules $[3,4,7]$, which is illustrated by (15)(17); namely, to "create" one kilowatt-hour at a point $p t u$ it is necessary to have $n$ kilowatt-hours at a point $p t d s$ and also a PTL connecting both points and able to transmit this amount of EP from ptds to $p t u$. Similar logics will be applied everywhere above to all components of ElcI and FI).

If a PTDS, located at a point $p t d s$, is connected to power terminal units, located at points $p t u_{1}, \ldots, p t u_{m}$, then this fragment of an ElcI is represented by $m$ following unitary rules:

$$
\begin{gathered}
\left(\boldsymbol{k W h}: p t u_{1}\right) \rightarrow n_{1} \cdot(\boldsymbol{k W h}: p t d s), n_{1} \cdot\left[p t d s, p t u_{1}\right], \\
\ldots \\
\left(\boldsymbol{k W h}: p t u_{m}\right) \rightarrow n_{m} \cdot(\boldsymbol{k W h}: p t d s), n_{m} \cdot\left[p t d s, p t u_{m}\right]
\end{gathered}
$$

Similarly may be represented fragments of an ElcI, consisting of connected PTDSs. In this case a string $p t d s$ is a representation of a location of a delivering power transforming-distributing substation, whilst $p t d s_{1}, \ldots, p t d s_{l}$ - locations of such PTDSs, which are consuming power transformed and transmitted by it:

$$
\begin{gathered}
\left(\left(\boldsymbol{k W h}: p t d s_{1}\right) \rightarrow n_{1} \cdot(\boldsymbol{k W h}: p t d s), n_{1} \cdot\left[p t d s, p t d s_{1}\right],\right. \\
\ldots \\
\left(\boldsymbol{k W h}: p t d s_{l}\right) \rightarrow n_{l} \cdot(\boldsymbol{k W h}: p t d s), n_{l} \cdot\left[p t d s, p t d s_{l}\right]
\end{gathered}
$$

In such a way all tree-like fragments of an ElcI are represented, until a power plant, producing electric power. Any tree-like fragment of an ElcI, containing some PP and connected with it PTDSs, may be represented by following URs:

$$
\begin{aligned}
\left(\boldsymbol{k W h}: p t d s_{1}\right) & \rightarrow n_{1} \cdot(\boldsymbol{k W h}: p p), n_{1} \cdot\left[p p, p t d s_{1}\right], \\
\left(\boldsymbol{k W h}: p t d s_{l}\right) & \rightarrow n_{l} \cdot(\boldsymbol{k W h}: p p), n_{l} \cdot\left[p p, p t d s_{l}\right],
\end{aligned}
$$

and, if there are some power terminal units connected to a power plant directly, i.e. without any intermediate PTDSs, then also

$$
\begin{gathered}
\left(\boldsymbol{k W h}: p t u_{1}\right) \rightarrow n_{1} \cdot(\boldsymbol{k W h}: p p), n_{1} \cdot\left[p p, p t u_{1}\right], \\
\ldots \\
\left(\boldsymbol{k W h}: p t u_{m}\right) \rightarrow n_{m} \cdot(\boldsymbol{k W h}: p p), n_{m} \cdot\left[p p, p t u_{m}\right],
\end{gathered}
$$

where $p p$ is a location of a power plant.

A power plant, in turn, may be represented by an UR

$$
(\boldsymbol{k W h}: p p) \rightarrow n_{1} \cdot\left(\operatorname{res}_{1}: p_{1}\right), \ldots, n_{k} \cdot\left(\operatorname{res}_{k}: p_{k}\right)
$$


where $n_{1}, \ldots, n_{k}$ are amounts of resources res $_{1}, \ldots$, res $_{k}$, which must be delivered to locations $p_{1}, \ldots, p_{k}$ respectively in order to generate one kilowatt-hour of electric power at a location $p p$, from which, in turn, it may be delivered by PTLs to PTDSs (PTUs), closest to a PP.

By this, evidently, $p_{1}, \ldots, p_{k}$ are locations of terminal units of a fuel infrastructure, which, in turn, delivers the aforementioned resources - most frequently, natural gas and various oil derivatives, which are transferred to power plants by pipelines, as it was described in the Section II.

Fuel terminal units, delivering resources to consumers, are represented as headers of unitary rules of the form

$$
(\text { res: } f t u) \rightarrow n \cdot(\text { res: } f d s), m \cdot(\boldsymbol{k W h}: p t u), n \cdot[f d s, f t u],
$$

where multiobject $m \cdot(\boldsymbol{k} \boldsymbol{W h}: p t u)$ represents a PTU of an electricity infrastructure, located at a point $p t u$ and providing operation of an FTU located at a point $f t u$ during delivery of one unit of a resource res from a point $f d s$ to a point $f t u$. This amount of power is consumed during a resource transfer via a pipe, which start point is $f d s$ and final point is $f t u$. In a general case, due to losses of fuel during it's transfer via a pipe, $n \geq 1$ units of fuel are needed to be delivered to a pump at a start point of this pipe.

Distributing facilities (namely, FDSs) of fuel infrastructure may be represented similarly to PTDSs:

$$
\begin{aligned}
& \left(\text { res: } f d s_{1}\right) \rightarrow n_{1} \cdot(\text { res: } f d s), m_{1} \cdot\left(\boldsymbol{k W h}: p t u_{1}\right), n_{1} \cdot\left[f d s, f d s_{1}\right], \\
& \left(\text { res: } f d s_{k}\right) \rightarrow n_{k} \cdot(\text { res: } f d s), m_{k} \cdot\left(\boldsymbol{k W h}: p t u_{k}\right), n_{k} \cdot\left[f d s, f d s_{k}\right] \\
& \left(\text { res: } f t u_{1}\right) \rightarrow n_{1}^{\prime} \cdot(\text { res: } f d s), m_{1}^{\prime} \cdot\left(\boldsymbol{k W h}: p t u_{1}^{\prime}\right), n_{1}^{\prime} \cdot\left[f d s, f t u_{1}\right], \\
& \left(\text { res: } f t u_{l}\right) \rightarrow n_{l}^{\prime} \cdot(\text { res: } f d s), m_{l}^{\prime} \cdot\left(\boldsymbol{k W h}: p t u_{l}^{\prime}\right), n_{l}^{\prime} \cdot\left[f d s, f t u_{l}\right],
\end{aligned}
$$

that means that delivered resource, incoming to any FDS, is distributed to $k+l$ pipes by application of corresponding needed amounts of electric power. The first $k$ pipes provide fuel transfer to another FDSs whilst the last $l$ - to FTUs. As above, $\left[f d s, f t u_{i}\right], i=1, \ldots, l$, are pipes, which start point is $f d s$ and final points are $f t u_{i}$. Similarly, $\left[f d s, f d s_{j}\right], j=1, \ldots, k$, are pipes, which start point is $f d s$ and final points are $f d s_{j}$. Presence of objects $\left(\boldsymbol{k W h}: p t u_{i}\right)$ in all unitary rules (36) and objects $\left(\boldsymbol{k W h}: p t u_{j}^{\prime}\right)$ in all unitary rules (37) means that power terminal units, belonging to an electricity infrastructure, would be installed and operate at some predefined points $p t u_{i}$ and $p t u_{j}^{\prime}$ respectively to make possible physical contact with FDSs and FTUs and their power supply during transfer of resource res.

As it was mentioned above, in a general case every pipe has it's own technical parameters - finally, it's own amounts of electric power consumed, i.e. $m_{i}$ and $m_{j}^{\prime}$, as well as losses of a fuel during it's transfer via this pipe, i.e. $n_{i}$ and $n_{j}^{\prime}$.

As it is clear, the described techniques may be applied until places of origination of energy carriers, i.e. fuel production plants, working out pipeline gas and various oil derivatives, used as a fuel by power plants. As it was assumed above, PECs, used for fuel production, are accumulated at fuel storages collocated with FPPs. So operation of any such FPP may be represented as follows:

$$
\begin{gathered}
(\text { res: fpp }) \rightarrow \\
n \cdot(\boldsymbol{k W h}: p t u), \\
m_{1} \cdot\left(\text { res }_{1}: f s_{1}\right), n_{1} \cdot\left(\boldsymbol{k W h}: p t u_{1}\right), \\
\ldots, \\
m_{t} \cdot\left(\operatorname{res}_{t}: f s_{t}\right), n_{t} \cdot\left(\boldsymbol{k W h}: p t u_{t}\right)
\end{gathered}
$$


where $f s_{1}, \ldots, f s_{t}$ are points, where fuel storages with PECs $r e s_{1}, \ldots, r e s_{t}$ are located, so namely regarding these places power terminal units would be installed, thus providing relocation of amounts of these PECs necessary to an FPP for production of one unit of fuel res at a location $f p p$. The aforementioned relocation would be possible if needed amounts of electric power, i.e. $n_{1}, \ldots, n_{t}$ kilowatthours, would be available at points $p t u_{1}, \ldots, p t u_{t}$ where respective PTUs are operating. In turn, to produce one unit of a fuel res an FPP itself would consume $n$ kilowatt·hours from a power terminal unit located at a point $p t u$.

One more nuance connected with a multigrammatical representation of an energy infrastructures and assessment of their resilience is representation of active states of EI elements. To represent the fact that any producing or transmitting (transferring) facility (PP, FTP, PTDS, FTDS, PTU, FTU) to carry out it's functions would be in an active state we shall apply techniques proposed and described in $[3,4,7]$ regarding industrial systems and based on inclusion to bodies of unitary rules special multiobjects. So in the case of URs (24)-(37) concerning ElcI any unitary rule

$$
(\boldsymbol{k W h}: x) \rightarrow X
$$

where $X$ is a body of this UR, would be transformed to

$$
(\boldsymbol{k W h}: x) \rightarrow X, 1 \cdot(+x),
$$

where symbol " + " means that a facility $x$ is in an active state and may produce one kilowatt·hour of an EP. Similarly, unitary rules (35)-(38) concerning FI

$$
(\text { res }: x) \rightarrow X
$$

would be transformed to

$$
(\text { res: } x) \rightarrow X, 1 \cdot(+x)
$$

This means that a facility $x$ is in an active state and may produce one unit of a resource res. Following $[3,4,7]$, we shall use below the notion "operation cycle of a facility $x$ " (for short OCF), understanding it as an action performed by a facility to produce one unit of EP, fuel or some other resource. A set (not obligatory a sequence) of $l$ such OCFs inside a considered time period of an EI operation has an evident representation by a multiobject $l \cdot(+x)$.

We shall denote a set of unitary rules representing ElcI, FI and their interconnections, as described above, by $R_{E}$. Let us illustrate techniques of construction such set given a graph representation of an EI.

Example 4. Consider the EI segment represented by the graph at Fig. $1 a$ and Table 1 . It may be also represented as a following set of unitary rules:

$(\boldsymbol{k W h}:$ Ptds1) $\rightarrow 1 \cdot(\boldsymbol{k W h}: P p), 1 \cdot[$ Pp,Ptds1] $1 \cdot(+P t d s 1)$

$(\boldsymbol{k W h}:$ Ptds2) $\rightarrow 1 \cdot(\boldsymbol{k W h}:$ Pp $), 1 \cdot[$ Pp,Ptds2] $1 \cdot(+P t d s 2)$;

$($ kWh:Ptu1) $\rightarrow 1 \cdot($ kWh:Ptds1), $1 \cdot[$ Ptds1,Ptu1 $], 1 \cdot(+P t u 1)$;

$($ kWh:Ptu2) $\rightarrow 1 \cdot($ kWh:Ptds1), $1 \cdot[$ Ptds1,Ptu2], $1 \cdot(+P t u 2)$;

$($ kWh:Ptu3 $) \rightarrow 1 \cdot($ kWh:Ptds1), $1 \cdot[$ Ptds1,Ptu3], $1 \cdot(+P t u 3)$;

$($ kWh:Ptu4 $) \rightarrow 1 \cdot($ kWh:Ptds 2$), 1 \cdot[$ Ptds 2, Ptu4 $], 1 \cdot(+P t u 4)$

$($ kWh:Ptu5) $\rightarrow 1 \cdot($ kWh:Ptds2), $1 \cdot[$ Ptds 2,Ptu5], $1 \cdot(+P t u 5)$;

$($ kWh:Ptu6) $\rightarrow 1 \cdot($ kWh:Ptds2), $1 \cdot[$ Ptds2,Ptu6], $1 \cdot(+P t u 6)$;

$($ kWh:Ptu7 $) \rightarrow 1 \cdot($ kWh:Ptds 1), $1 \cdot[$ Ptds 1,Ptu7], $1 \cdot(+P t u 7)$;

$($ kWh:Ptu8) $\rightarrow 1 \cdot($ kWh:Ptds2), $1 \cdot[$ Ptds 2, Ptu8 $], 1 \cdot(+P t u 8)$; 
$($ kWh:Ptu9 $) \rightarrow 1 \cdot($ kWh:Ptds2), $1 \cdot[$ Ptds2,Ptu9], $1 \cdot(+$ Ptu9 $)$;

$($ kWh:Pp $) \rightarrow 3 \cdot($ TonFuel: Ftu1) $) 1 \cdot[$ Ftu1, Pp $], 1 \cdot(+P p)$;

$($ TonFuel: Ftu1) $\rightarrow 1.05 \cdot($ TonFuel:Fds2), $20 \cdot($ kWh:Ptu1), $1 \cdot[$ Fds 2, Ftu1 $], 1 \cdot(+$ Ftu1) ;

$($ TonFuel:Ftu2) $\rightarrow 1.01 \cdot($ TonFuel:Fds1), $20 \cdot($ kWh:Ptu2 $), 1 \cdot[$ Fds $2, F t u 2], 1 \cdot(+F t u 2)$;

$($ TonFuel: Ftu3) $\rightarrow 1.02 \cdot($ TonFuel: Fds1), $20 \cdot($ kWh:Ptu2 $), 1 \cdot[$ Fds2,Ftu3], $1 \cdot(+$ Ftu3) ;

(TonFuel:Fds2) $\rightarrow 1.01 \cdot($ TonFuel:Fds1), $30 \cdot(\mathrm{kWh}:$ Ptu2 $), 1 \cdot[$ Fds1,Fds2],1·(+Fds2) ;

(TonFuel: Fds1) $\rightarrow 1.01 \cdot($ TonFuel: Fpp), $40 \cdot($ kWh:Ptu1), $1 \cdot[$ Fpp, Fds1], $1 \cdot(+F d s 1)$;

$($ TonFuel: Fpp $) \rightarrow 2.9 \cdot($ TonCrudeOil: Fs $), 50 \cdot($ kWh:Ptu4 $), 1 \cdot[$ Fs, Fpp $], 1 \cdot(+F p p)$.

As seen, the URs (43) - (44) represent knowledge about the PTDSs "1" and "2", which are located

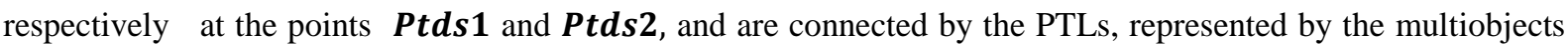
$1 \cdot[\boldsymbol{P p}, \boldsymbol{P t d s} 1]$ and $1 \cdot[\boldsymbol{P P}, \boldsymbol{P t d s} 2]$, with the power plant located at the place $\boldsymbol{P p}$; there are no valuable losses of the EP during it's transmission from the PP to both PTDSs, so the same amount of the EP which is given into any PTL by the PP is received by a PTDS; hence, the multiplicities of the object ( $\boldsymbol{k} \boldsymbol{W} \boldsymbol{h}: \boldsymbol{P p})$ in both URs (43) and (44) are equal to 1 . The multiobjects $1 \cdot(+$ Ptds1) and $1 \cdot(+$ Ptds 2$)$ represent a fact that both PTDSs would be in active states to receive the EP from the producing it power plant and to deliver the EP to the connected with them power terminal units or PTDSs. The knowledge about PTUs "1" - "9", connected with the respective PTDSs in full accordance with the graph representation of the considered segment of the EI, is represented by the URs (45) - (53). The UR (54) represents, that the power plant may produce one kilowatt hour consuming for this objective 3 tons of the fuel (represented by the multiobject $3 \cdot($ TonFuel:Ftu1)), receiving it via the pipe (represented by the MO $1 \cdot[\boldsymbol{F t u 1}, \boldsymbol{P p}]$ ) from the fuel terminal unit "1" located at the point Ftu1, and being in the active state, that is represented by the MO $1 \cdot(+\boldsymbol{P p})$. The URs (55)-(57) represent knowledge about the fuel terminal units "1" - "3". The UR (55) represents the knowledge about the resources necessary to

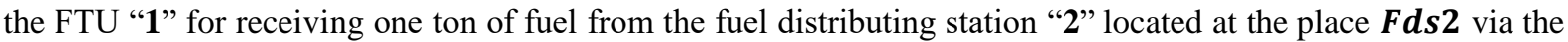
pipe represented by the MO $1 \cdot[\boldsymbol{F d s} \mathbf{2}, \boldsymbol{F t u 1}]$. Due to the fuel losses during transfer, the FDS " $\mathbf{2}$ ", delivering the fuel to the FTU "1", gives into the pipe, represented by the MO $1 \cdot[\boldsymbol{F d s} 2, \boldsymbol{F t u 1}], 1.05$ ton of the fuel, that is represented by the MO 1.05 (TonFuel:Fds2). The FTU "1" to receive one ton of the fuel consumes 20 kilowatthours of the EP from the power terminal unit located at the point Ptu1, that is represented by the MO $20 \cdot(\boldsymbol{k W h}: \boldsymbol{P t u 2})$. And, as usual, the FTU "1" must be in the active state, that is represented by the MO $1 \cdot(+$ Ftu 1$)$. The URs (56)-(57) in the same manner represent the knowledge about the fuel terminal units "2" and " 3 " which are provided by the EP from the PTU "2", and this PTU consumes the same 20 kilowatt-hours for one ton of the received fuel. The URs (58)-(59) represent the similar knowledge about the fuel distributing stations "1" and " 2 " provided by the EP from the PTUs " 3 " and " 2 " respectively; the FDS " 1 " consumes 40 kilowatthours of EP from the PTU "7" located at the point Ptu3, that is represented by the MO $40 \cdot(\boldsymbol{k W h}: \boldsymbol{P t u 3})$, and the FDS"2" consumes 30 kilowatt-hours of EP from the PTU "2", located at the point $\boldsymbol{P t u 2}$, that is represented by the MO $30 \cdot(\boldsymbol{k W h}: \boldsymbol{P t u 2})$. The FDS "2" receives the fuel from the FDS "1" via the pipe represented by the MO $1 \cdot[\boldsymbol{F d s} \mathbf{1}, \boldsymbol{F d s} 2]$. The FDS "1", in turn, receives the fuel from the fuel producing plant via the pipe represented by the MO $1 \cdot[\boldsymbol{F p p}, \boldsymbol{F d s} \mathbf{1}]$ consuming 40 kilowatthours of EP from the PTU "3", located at the point Ptu3, and this is represented by the MO $40 \cdot(\boldsymbol{k W h}: \boldsymbol{P t u 3})$. At last, the UR (60) represents the knowledge about the FPP which is capable to produce one ton of the fuel receiving 2.9 tons of crude oil from the fuel storage via a pipe represented by the MO $1 \cdot[\boldsymbol{F} \boldsymbol{s}, \boldsymbol{F p p}]$ and consuming 50 kilowatthours of EP from the PTU "4", located at the point Ptu4, and this is represented by the MO $50 \cdot(\boldsymbol{k W h}:$ Ptu4 $)$. Finally, as seen, the considered segment of the EI, consuming crude oil from the fuel storage, provides external consumers by the electric power and the fuel, respectively, via the PTUs " 5 ", " 6 " and "7", and via the FTUs " 2 " and "3".

A resource base of any EI may be represented as a multiset $v_{E}$ including multiobjects of the following three types: 
1) $m \cdot($ res $: p)$ for all fuel storages entering a considered EI, that means $m$ units of materiel resource (PEC or produced fuel) res are available at some FS located at a place $p$;

2) $N \cdot\left[p, p^{\prime}\right]$ for all links having place in a considered EI, that means a value $N$ is a bandwidth (throughput capacity) of a link $\left[p, p^{\prime}\right]$, i.e. a maximal amount of EP or materiel resource, which may be transmitted (transferred) via this link during a considered time period (in the case $\left[p, p^{\prime}\right]$ is a PTL this amount is measured in kilowatthours whilst in the case $\left[p, p^{\prime}\right]$ is a pipe this amount may be measured in barrels, cubic meters, kilograms, tons etc.);

3) $L \cdot(+x)$ for all elements of a considered EI, thus establishing for any such element a maximal number of operation cycles which might be executed by it at a considered time period (in other words, $L$ is fixing a maximal productivity of an element $x$; a multiobject $L \cdot(+x)$ will be referred below as an operation resource of an element $x$ ).

So in fact a resource base of any EI includes not only materiel resources (primary and produced energy carriers), but also operation resources of it's elements, as well as throughput capacities of it's links.

Example 5. The resource base of the segment of the EI considered in the previous Example 4 and corresponding to the knowledge represented by the Table 1, is as follows:

$$
\begin{aligned}
& v_{E}= \\
& \{100 \cdot(\text { TonCrudeOil: Fs), } 1000 \cdot[\text { Pp, Ptds1], } 1100 \cdot[\text { Pp,Ptds2], } 200 \cdot[\text { Ptds1,Ptu1], } \\
& 300 \cdot[\text { Ptds1, Ptu2], } 400 \cdot[\text { Ptds1, Ptu3], } 100 \cdot[\text { Ptds1, Ptu7], 200 · Ptds2, Ptu4], } \\
& 300 \cdot[\text { Ptds2,Ptu5] , } 300 \cdot[\text { Ptds2,Ptu6], } 200 \cdot[\text { Ptds2,Ptu8], 100 } \cdot \text { [Ptds2,Ptu9], } \\
& 200 \cdot[\text { Fs, Fpp }], 200 \cdot[\text { Fpp }, F d s 1], 100 \cdot[\text { Fds1, Fds2], } 50 \cdot[\text { Fds1, Ftu2 }], 50 \cdot[\text { Fds1, Ftu }] \text {, } \\
& 100 \cdot[\text { Fds } 2, F t u 1], 100 \cdot[\text { Ftu1, Pp }], \quad 100 \cdot(+P p), 100 \cdot(+ \text { Ptds1 }), 100 \cdot(+P t d s 2), \\
& 100 \cdot(+ \text { Ptu1), } 100 \cdot(+ \text { Ptu2 }), 100 \cdot(+ \text { Ptu3 }), 100 \cdot(+ \text { Ptu4 }), 100 \cdot(+ \text { Ptu5), } 100 \cdot(+ \text { Ptu6), } \\
& 100 \cdot(+ \text { Ptu } 7), 10 \cdot(+ \text { Ftu1 }), 10 \cdot(+ \text { Ftu } 2), 10 \cdot(+ \text { Ftu } 3), 10 \cdot(+F d s 2), 10 \cdot(+F d s 1), \\
& 10 \cdot(+\boldsymbol{F p p})\} \text {. }
\end{aligned}
$$

As seen, the fuel storage entering the considered segment of an EI contains 100 tons of crude oil; the PTL connecting the power plant and the PTDS " 1 " during a considered time period provides transmission no more than 1000 kilowatt hours of EP that is represented by the MO $1000 \cdot[\boldsymbol{P p}, P t d s 1]$; the PTL connecting the PP and the PTDS "2" provides transmission no more than 1100 kilowatthours of EP that is represented by the MO $1100 \cdot[\boldsymbol{P p}, \boldsymbol{P t d s} 2]$; similarly are represented the upper threshold values of bandwidths of all other PTLs of the considered segment of the EI. The pipe, connecting the fuel storage and the fuel producing plant, provides delivery of no more than 200 tons of crude oil that is represented by the MO $200 \cdot[\boldsymbol{F s}, \boldsymbol{F p p}]$; the pipe, connecting the fuel producing plant and the fuel distributing station "1", provides delivery of no more than 200 tons of the fuel that is represented by the MO $200 \cdot[\boldsymbol{F p p}, \boldsymbol{F d s} \mathbf{s}]$; similarly are represented the upper threshold values of throughput capacities of all other pipes of the considered segment of the EI. Any element of the ElcI, entering this segment, during a considered period of time may execute 100 operation cycles, that is represented by the MOs $100 \cdot(+\boldsymbol{P p}), \ldots, 100 \cdot(+\boldsymbol{P t u} 9)$; any element of the FI, entering this segment, during a considered period of time may execute 10 operation cycles, that is represented by the multiobjects $10 \cdot(+\boldsymbol{F t u 1}), \ldots, 10 \cdot(+\boldsymbol{F p p})$.

After specifying a resource base, an EI $E$ may be considered as a free industrial system $\left.E=<\{\varnothing\}, R_{E}, v_{E}\right\rangle$ in the sense [7]. Similarly, a demand on electric power and fuel (an order to be completed in the sense of [7]) may be represented as a multiset $q_{E}$ containing multiobjects like $n \cdot(\boldsymbol{k W} \boldsymbol{h}: p)$, representing $n$ kilowatt-hours which would be delivered to a consumer located at a place $p$ where some PTU providing this delivery is located, and multiobjects like $m \cdot($ res: $p$ ), representing $m$ units of a fuel (or any other materiel resource) res which would be delivered to a consumer located at a place $p$ where an FTU providing this delivery is located. As a result, an EI providing delivery of needed to consumers amounts of power and fuel may be considered as an 
industrial system $E_{q}=<q, R_{E}, v_{E}>$ assigned to an order $q$ in the sense [7]. Following [7], this representation of an IS implies a filtering unitary multiset grammar $S_{q}=<q, R_{E}, F_{E}>$, where

$$
F_{E}=\left\{a \leq n \mid n \cdot a \in v_{E}\right\} \cup\left\{a=0 \mid a \in \bar{A}_{S} \& a \bar{\epsilon} v_{E}\right\},
$$

in such a way that this FUMG generates a set of terminal multisets each representing some collection of resources sufficient for an order $q$ completion by some definite cooperation of manufacturing devices (the second operand of a join is obligatory to eliminate ways of an order completion which satisfy restrictions implied by an available resource base of an EI, but need some additional resources which are absent at an RB at all).

As now may be seen, a unitary multiset grammar $S_{q}=<q, R_{E}>$ defines a set $\bar{V}_{S_{q}}$ of terminal multisets each having a form

$$
\begin{gathered}
\left\{M_{1} \cdot\left(\operatorname{res}_{i_{1}}: p_{i_{1}}\right), \ldots, M_{s} \cdot\left(\operatorname{res}_{i_{t}}: p_{i_{t}}\right), N_{1} \cdot\left[p_{j_{1}}, p^{\prime}{ }_{j_{1}}\right], \ldots, N_{u} \cdot\left[p_{j_{u}}, p_{j_{u}}^{\prime}\right]\right. \\
\left.L_{1} \cdot\left(+x_{k_{1}}\right), \ldots, L_{z} \cdot\left(+x_{k_{z}}\right)\right\}
\end{gathered}
$$

where $M_{1}, \ldots, M_{s}$ are amounts of, respectively, resources $\operatorname{res}_{i_{1}}, \ldots$, res $_{i_{t}}$ (PECs stored at FSs, fuels, produced by FPPs, as well as EP, produced by PPs) to be available at places $p_{i_{1}}, \ldots, p_{i_{t}}$ (via PTUs, FTUs, or directly from fuel storages); $N_{1} \ldots, N_{u}$ are amounts of energy carriers and electric power to be transferred (transmitted) via, respectively, links $\left[p_{j_{1}}, p_{j_{1}}^{\prime}\right], \ldots,\left[p_{j_{u}}, p_{j_{u}}^{\prime}\right]$ (PTLs and pipes) during a considered time period; $L_{1} \ldots, L_{z}$ are numbers of operation cycles of, respectively, facilities $x_{k_{1}}, \ldots, x_{k_{z}}$ involved in a completion of an order $q$. So every TMS $v \in \bar{V}_{S_{q}}$ corresponds to some specific way of an order $q$ completion (in a general case there may be several ways identical by resource consumption and facilities involvement).

We shall represent an EI current resource base $v_{E}$ as a sum of three multisets

$$
v_{E}=v_{E}^{r e s}+v_{E}^{p}+v_{E}^{x}
$$

the first

$$
v_{E}^{r e s}=\left\{\boldsymbol{M}_{1} \cdot\left(\operatorname{res}_{\boldsymbol{i}_{\mathbf{1}}}: p_{\boldsymbol{i}_{\mathbf{1}}}\right), \ldots, \boldsymbol{M}_{\boldsymbol{s}} \cdot\left(\operatorname{res}_{\boldsymbol{i}_{\boldsymbol{t}}}: p_{\boldsymbol{i}_{\boldsymbol{t}}}\right)\right\}
$$

representing amounts of resources having place at an EI fuel storages, the second

$$
v_{E}^{p}=\left\{\boldsymbol{N}_{1} \cdot\left[p_{\boldsymbol{j}_{1}}, p_{\boldsymbol{j}_{\mathbf{1}}}^{\prime}\right], \ldots, \boldsymbol{N}_{\boldsymbol{u}} \cdot\left[p_{\boldsymbol{j}_{\boldsymbol{u}}}, p_{\boldsymbol{j}_{\boldsymbol{u}}}^{\prime}\right]\right\}
$$

representing current bandwidths and throughput capabilities of an EI links, and the third

$$
v_{E}^{x}=\left\{\boldsymbol{L}_{\mathbf{1}} \cdot\left(+x_{\boldsymbol{k}_{1}}\right), \ldots, \boldsymbol{L}_{\mathbf{z}} \cdot\left(+x_{\boldsymbol{k}_{\mathbf{z}}}\right)\right\}
$$

representing current operation resource of an EI facilities. (Bold indices $\boldsymbol{i}_{1}, \ldots, \boldsymbol{i}_{t}, \boldsymbol{j}_{1}, \ldots, \boldsymbol{j}_{\boldsymbol{u}}, \boldsymbol{k}_{\mathbf{1}}, \ldots, \boldsymbol{k}_{\mathbf{z}}$, used in (64) -(67), differ from ordinary indices $i_{1}, \ldots, i_{t}, j_{1}, \ldots, j_{u}, k_{1}, \ldots, k_{z}$, used in (63)).

\section{Cyclicity of FUMGs, representing energy infrastructures, and their finitarization}

Let us note, that industrial systems are represented through a technological interpretation of unitary rules $[3,4$, 7], or in other words, through their capability to manufacture (assemble) some complex objects from their 
components until some atomary (non-splitted) elements (spare parts, microchips, etc.); thus FUMGs representing ISs are essentially acyclic, and, hence, STMSs generated by their application, are finite.

Unlike industrial systems, energy infrastructures operate in such a way that it's fuel segment (namely, FI) consumes EP generated by it's electricity segment (namely, ElcI), whilst the last one consumes fuel necessary for EP production. Thus FUMGs representing EIs are essentially cyclic, and sets of multisets generated by their application are in a general case infinite: for a core UMG $S_{q}^{\prime}=<q, R_{E}>$ of a FUMG $S_{q}=<q, R_{E}, F_{E}>$ it would be valid

$$
\bar{V}_{S^{\prime} q}=\{\varnothing\}
$$

and, simultaneously,

$$
\left|V_{S^{\prime} q}\right|=\infty
$$

hence direct application of an ISs multigrammatical representation and criterial base to EIs is in fact impossible. So a task is to find such local correction of the aforementioned representation of ISs which provide finitarity of FUMGs representing EIs. Such correction will be called finitarization of FUMGs.

Here we propose a simple solution of this problem based on the so called terminalization of non-terminal objects introduced in [7] as a tool of modelling ISs, which resource bases contain not only primary (non-splitted) components of objects specified by an order to an IS, but also components, manufactured by an IS beginning from the aforementioned primary ones at previous steps of it's operation. Namely, we shall extend a set of URs $R_{E}$ in a following way. Let $R_{E}$ contains an unitary rule

$$
(\boldsymbol{k W h}: x) \rightarrow X,
$$

where $X$ is a non-empty body. We shall join to $R_{E}$ an unitary rule

$$
(\boldsymbol{k W h}: x) \rightarrow k \cdot\left(\text { res: } x^{\prime}\right)
$$

that means one kilowatthour would appear at a location $x$ as a result of consumption $k$ units of resource res located at a place $x^{\prime}$, and, that is most essential, $\left(\right.$ res: $\left.x^{\prime}\right)$ is a terminal object, that means $R_{E}$ does contain no one UR with a header (res: $x^{\prime}$ ); the last, in turn, means, that there is an alternative way of such appearance, not involving chain of mutual demands determined by a body $X$ of UR (70). In most cases such resource res is power, accumulated at previous steps of operation of an EI or generated by some initiating action or operation (for example, activation of a car ignition system).

In the first case power storages (PSs) similar to fuel storages are presumed, and, like FSs, they may be represented by multiobjects

$$
n \cdot(\boldsymbol{k W h}: x) \in v_{E}
$$

that means a PS located at a place $x$ may provide on demand up to $n$ kilowatt-hours.

The second case (power generation by some initiating action) is simply reduced to the first one by including to a resource base the same multiobject as in (72), that reflects an obstacle, that a source of the aforementioned action is, finally, is also some kind of a power storage.

Thus, introducing by (71)-(72) a concept of a power storage, which, in fact, fully reflects essence of real processes of power supply, we have proposed the simplest way of finitarization of FUMGs representing EIs. Now, evidently, despite a set $V_{S^{\prime} q}$ remains infinite, a set $\bar{V}_{S^{\prime} q}$ in a general case would be non-empty, thus representing at least one way of an order $q$ completion by application of a priori accumulated power; from the 
mathematical point of view, this means that a core UMG $S^{\prime}{ }_{q}=<q, R_{E}>$ of a FUMG $S_{q}=<q, R_{E}, F_{E}>$ generates at least one terminal TMS.

Now we are ready to consider the main result of this paper being a criterial base for the assessment of vulnerability of energy infrastructures to destructive impacts.

\section{Criteria of vulnerability of an energy infrastructure to a destructive impact}

Let us begin from the initial task, which verbal formulation is as follows: given amounts of primary energy carriers at fuel storages of an EI and demand on an electric power and fuels from it's external consumers (an order to be completed by an EI), to assess whether an EI is or is not capable to complete an order (i.e. to provide these consumers by required amounts of $\mathrm{EP}$ and fuels).

Due to the introduced techniques of EIs representation, now to solve this task it is sufficient to apply the criterion [7], proposed regarding industrial systems, to energy infrastructures.

Statement 1. An energy infrastructure $E=<\{\varnothing\}, R_{E}, v_{E}>$ is not capable to complete an assigned order $q$, if

$$
\left(\forall v \in \bar{V}_{S_{q}}\right) v_{E} \subset v
$$

where $S_{q}=<q, R_{E}>$

Speaking informally, an EI $E$ is not capable to complete an assigned order $q$, if there exists no one way of generation (production) and delivery of necessary amounts of EP and fuels, consuming for this objective such amounts of primary energy carriers which are not greater than available at fuel storages of this EI, and also capabilities of EI facilities and links are sufficient for these generation (production) and delivery. Following [7], this criterion may be represented by applying a respective filtering unitary multiset grammar $\left.S_{q}=<q, R_{E}, F_{E}\right\rangle$, where

$$
F_{E}=\left\{a \leq n \mid n \cdot a \in v_{E}\right\} \cup\left\{a=0 \mid a \in \overline{A_{S}} \& a \bar{\epsilon} v_{E}\right\}
$$

(the second operand of a join is obligatory to eliminate ways of order completion which satisfy restrictions implied by an available resource base of an EI, but need some additional resources which are absent at an RB at all).

Statement 2. Energy infrastructure $E=<\{\varnothing\}, R_{E}, v_{E}>$ is not capable to complete an assigned order $q$, if

$$
\bar{V}_{S_{q}}=\{\varnothing\}
$$

where $S_{q}=<q, R_{E}, F_{q}>$

All the said forms a background for the strict consideration of a task of an assessment of vulnerability of EIs to destructive impacts. Following [7], we shall represent an impact as a multiset $\Delta v$ which determines eliminated by this impact capabilities of an EI elements (facilities and links) and stored at FSs amounts of primary energy carriers. After such impact application a resource base $v_{E}$ of an EI becomes $v_{E}-\Delta v$. Such representation in a general case provides any possible variants of impact, which may destroy EI elements, reduce bandwidths (throughput capacities) of links PTLs and pipes, as well as reduce amounts of PECs in FSs.

Namely,

$$
m \cdot(r e s: p) \in \Delta v
$$

means, that an impact eliminates $m$ units of a resource from an FS located at a place $p$. Similarly,

$$
n \cdot\left[p, p^{\prime}\right] \in \Delta v
$$


means, that an impact reduces for $m$ units a maximal amount of EP or fuel which may be transmitted (transferred) at a considered time period via a PTL (pipe) with start point $p$ and final point $p^{\prime}$. Finally, to represent destruction of any producing or transmitting (transferring) facility (PP, FTP, PTDS, FTDS, PTU, FTU) we may apply the same techniques including to an MS $\Delta v$ multiobjects like $l \cdot(+x)$ representing that an element of EI would be affected, and a result of this action would be reduction of an operation resource of this element by $l$ units. Obviously, a case of an entire destruction of any component of an EI may be easily represented by inclusion to a multiset $\Delta v$ an object $\boldsymbol{N} \cdot(a)$, where $\boldsymbol{N}$ is a number maximal for the used implementation of FUMGs algorithmics, so for any $k$

$$
\{k \cdot(a)\}-\{\boldsymbol{N} \cdot(a)\}=\{\varnothing\}
$$

is valid.

Example 6. Let the destructive impact destroys facilities PTDS "1" and PTU "7" of the considered in the previous Example 5 segment of an EI as well as reduces amount of the crude oil at the fuel storage by 20 tons, and also reduces bandwidths (throughput capacities) of: PTL [Ptds2,Ptu4] by 100 kilowatt-hours, PTL [Ptds2,Ptu5] by 200 kilowatt-hours, and pipe $[\boldsymbol{F d s} 2, \boldsymbol{F t u 1}]$ by 10 tons of fuel. So the result of this impact will be

$$
\begin{gathered}
v_{E}-\Delta v= \\
v_{E}-\{10000 \cdot(+ \text { Ptds1 }), 10000 \cdot(+ \text { Ptu } 7), 20 \cdot(\text { TonCrudeOil: Fs }) \\
100 \cdot[\text { Ptds } 2, \text { Ptu }], 200 \cdot[\text { Ptds } 2, \text { Ptu } 5], 10 \cdot[\text { Fds } 2, \text { Ftu } 1]\}
\end{gathered}
$$

where $\boldsymbol{N}=10000$.

Let us begin from the simplest case when an impact is applied to an EI before a beginning of an order $q$ completion. Evidently, an impact $\Delta v$ transforms an EI $E=<\{\varnothing\}, R_{E}, v_{E}>$ to an affected EI $E=<\{\varnothing\}, R_{E}, v_{E}-\Delta v>$.

Now to obtain a necessary criteria of vulnerability of energy infrastructures to destructive impacts, it is sufficient to apply Statements 1 and 2 to an affected EI.

Statement 3. An energy infrastructure $E=<\{\varnothing\}, R_{E}, v_{E}>$ is vulnerable to an impact $\Delta v$, applied before a beginning of an assigned order $q$ completion, if

$$
\left(\forall v \in \bar{V}_{S_{q}}\right) v_{E}-\Delta v \subset v
$$

where $S_{q}=<q, R_{E}>$

Verbally, if no one way of an order $q$ completion is implementable (any way needs additional resources regarding available after an impact), then an energy infrastructure is vulnerable to an applied impact. Similarly to the Statement 3, this criterion may be represented by the application of FUMGs.

Statement 4. An energy infrastructure $E=<\{\varnothing\}, R_{E}, v_{E}>$ is vulnerable to an impact $\Delta v$, applied before a beginning of an assigned order $q$ completion, if

$$
\bar{V}_{S_{q}}=\{\varnothing\}
$$

where $S_{q}=<q, R_{E}, F_{E^{\prime}}>$, and

$$
F_{E^{\prime}}=\left\{a \leq n \mid n \cdot a \in v_{E}-\Delta v\right\} \cup\left\{a=0 \mid a \in \overline{A_{S}} \& a \bar{\epsilon} v_{E}-\Delta v\right\}
$$

Let us consider now a more general case, when an impact is applied to an EI at some time moment inside time period of an order completion. 
Just to this moment some part $\Delta q$ of an order $q$ may be completed, as well as a respective part $\Delta v_{E}$ of an EI resource base would be already consumed. If so, then there is not difficult to formulate statements being a corollaries of the Statements 3 and 4 and representing criteria of vulnerability of an EI affected by a destructive impact inside a time period of an order completion.

Statement 5. An energy infrastructure $E=<\{\varnothing\}, R_{E}, v_{E}>$ is vulnerable to an impact $\Delta v$, applied inside a time period of a completion an assigned order $q$, when a part $\Delta q$ of this order is already completed and a part $\Delta v_{E}$ of an EI resource base is already consumed, if

$$
\left(\forall v \in \bar{V}_{S_{q-\Delta q}}\right) v_{E}-\Delta v_{E}-\Delta v \subset v
$$

Statement 6. An energy infrastructure $E=<\{\varnothing\}, R_{E}, v_{E}>$ is vulnerable to an impact $\Delta v$, applied inside a time period of a completion an assigned order $q$, when a part $\Delta q$ of this order is already completed and a part $\Delta v_{E}$ of an EI resource base is already consumed, if

$$
\bar{V}_{S_{q-\Delta q}}=\{\varnothing\}
$$

where $S_{q-\Delta q}=<q, R_{E}, F_{E^{\prime \prime}}>$, and

$$
F_{E \prime \prime}=\left\{a \leq n \mid n \cdot a \in v_{E}-\Delta v_{E}-\Delta v\right\} \cup\left\{a=0 \mid a \in \overline{A_{S}} \& a \bar{\epsilon} v_{E}-\Delta v_{E}-\Delta v\right\}
$$

Now we may consider a more complicated case of EIs which topology is designed and resource base is maintained in such a way that if some destructive impact is applied before or during order completion, and this impact makes an EI not capable to complete this order, then an EI recovers itself by activation some prepared in advance amounts of operation resources as well as by application some prestored amounts of materiel resources.

\section{Modelling reservation and recovery of energy infrastructures}

Taking into account a possibility of application of destructive impacts of various nature to components of energy infrastructure, an EIs' management is usually preparing in advance some additional reserved facilities and primary or produced resources, which are made available promptly after an impact is detected, and such measure in many cases provides as effective as possible mitigation of consequences of the aforementioned impact (up to making this order feasible by the adjusted itself EI). A background of such adaptability is some redundancy implanted to an EI before or during it's operation [30-34]. The most usual measure implemented by EIs' designers and management are the so called backup power systems, providing EP generation for a time periods when the affected segments of EIs are recovered, and also bypasses, providing electricity or fuel flows by some workarounds if a preordered routes are broken by an impact.

It is not so difficult to apply the UMGs to represent the described opportunity. Namely, it is sufficient to join to an initial set of URs, representing a topology of an electricity infrastructure, unitary rules reflecting an alternative ways of EP transmission.

Namely, for any UR (18) representing a PTU, located at a point $p t u$, it is sufficient to join to a set $R_{E}$ one more UR

$$
(\boldsymbol{k W h}: p t u) \rightarrow n^{\prime} \cdot\left(\boldsymbol{k W h}: p t d s^{\prime}\right), n^{\prime} \cdot\left[p t d s^{\prime}, p t u\right]
$$

representing a fact that this PTU may receive EP not only from a PTDS located at a point ptds, but also from a PTDS located at a point $p t d s^{\prime}$. (It is assumed that there is a technological solution providing such opportunity). In a general case there may be $m \geq 1$ such alternative PTDSs capable to deliver EP to this PTU, and this is possible regarding any PTU entering a considered EI. 
Similarly, the same technique may be applied to PTDSs. To any UR entering a set (20) and representing a PTDS, located at a point $p t d s_{i}$, it is sufficient to join to a set $R_{E}$ one more UR

$$
\left(\boldsymbol{k W h}: p t d s_{i}\right) \rightarrow n_{i}^{\prime} \cdot\left(\boldsymbol{k W h}: p t d s^{\prime}\right), n_{i}^{\prime} \cdot\left[p t d s^{\prime}, p t d s_{i}\right]
$$

representing a fact that this PTDS may receive EP not only from a PTDS located at a point $p t d s$, but also from a PTDS located at a point $p t d s^{\prime}$. As in the case of PTUs, there may be $m \geq 1$ such alternative PTDSs capable to deliver EP to this PTDS, and this is possible regarding any PTDS entering a considered EI.

The described technique without any changes may be applied also to PTUs and PTDSs, connected directly with additional (reserve) power plants:

$$
\begin{aligned}
& \left(\boldsymbol{k W h}: p t d s_{j}\right) \rightarrow n_{j} \cdot\left(\boldsymbol{k W h}: p p^{\prime}\right), n_{j} \cdot\left[p p^{\prime}, p t d s_{j}\right] \\
& \left(\boldsymbol{k W h}: p t u_{k}\right) \rightarrow n_{k} \cdot\left(\boldsymbol{k W h}: p p^{\prime \prime}\right), n_{k} \cdot\left[p p^{\prime \prime}, p t u_{k}\right]
\end{aligned}
$$

These URs represent the facts, that a PTDS, located at a point $p t d s_{j}$, may receive EP not only from a PP located at a point $p p$, as defined by a respective UR from a set (21), but also from a PP located at a place $p p^{\prime}$, as well as PTU, located at a point $p t u_{k}$ and entering a set of URs (22), may receive EP from some PP located at a place $p p^{\prime \prime}$, which may differ from $p p^{\prime}$ or be the same. As in all considered above cases, there may be $m \geq 1$ such alternative power plants capable to deliver EP to these PTUs and PTDSs, and this is possible regarding any PTU and PTDS connected with several power plants.

Any reserve power plant, entering a considered ElcI and located at a point $p p^{\prime}$, may be represented by an UR

$$
\left(\boldsymbol{k W h}: p p^{\prime}\right) \rightarrow n_{1}^{\prime} \cdot\left(\operatorname{res}^{\prime}{ }_{1}: p^{\prime}{ }_{1}\right), \ldots, n_{k^{\prime}}^{\prime} \cdot\left(\operatorname{res}_{k^{\prime}}{ }^{\prime} p_{k^{\prime}}^{\prime}\right)
$$

where, as in (23), $n_{1}^{\prime}, \ldots, n^{\prime}{ }_{k^{\prime}}$ are amounts of resources $r e s^{\prime}{ }_{1}, \ldots, r e s^{\prime}{ }_{k^{\prime}}$, which must be delivered to locations $p^{\prime}{ }_{1}, \ldots, p_{k^{\prime}}^{\prime}$ respectively in order to generate one kilowatt-hour of electrical power at a location $p p^{\prime}$, from which, in turn, it may be delivered by PTLs to PTDSs (PTUs), closest to this PP. Let us note, that a reservation may be implemented not only by inclusion to an ElcI some additional power plants but also by implementation of alternative ways of EP generation and associated with them resources, by which a PP must be supplied. In this case to an UR (23) a unitary rule

$$
(\boldsymbol{k W h}: p p) \rightarrow n_{1}^{\prime} \cdot\left(\operatorname{res}_{1}^{\prime}: p_{1}^{\prime}\right), \ldots, n_{k^{\prime}}^{\prime} \cdot\left(\operatorname{res}_{k^{\prime}}: p_{k^{\prime}}^{\prime}\right)
$$

with the same header ( $\boldsymbol{k W h}: p p)$ and alternative body, representing a respective supply set, necessary for this way implementation, is joined to a set $R_{E}$.

As may be seen, due to an application of alternating UMGs it is quite easy to represent electric grids of any complexity, not only of tree-like structure, as it was considered above in the Section 4.

Similarly may be represented reservation of a fuel infrastructure.

Namely, for any UR (24) representing a FTU, located at a point $f t u$, it is sufficient to join to a set $R_{E}$ one more UR

$$
(\text { res: } f t u) \rightarrow n^{\prime} \cdot\left(\text { res: } f d s^{\prime}\right), m^{\prime} \cdot(\boldsymbol{k W h}: f t u), n \cdot\left[f d s^{\prime}, f t u\right],
$$

representing a fact that this FTU may receive a resource res not only from a FDS located at a point $f d s$, but also from a FDS located at a point $f d s^{\prime}$. (As in the case of ElcI, it is assumed that there is a technological solution providing such opportunity). In a general case there may be $m \geq 1$ such alternative FDSs capable to deliver a resource res to this FTU, and this is possible regarding any FTU entering a considered FI. 
Reservation of distributing facilities (namely, FDSs) of fuel infrastructure may be represented similarly to reservation of TDSs:

$$
\left(\text { res: } f d s_{i}\right) \rightarrow n_{i}^{\prime} \cdot\left(\text { res: } f d s^{\prime}\right), m_{1}^{\prime} \cdot\left(\boldsymbol{k W h}: p t u_{i}\right), n^{\prime}{ }_{1} \cdot\left[f d s^{\prime}, f d s_{i}\right]
$$

Any such UR represents the fact, that an FDS, located at a point $f d s_{i}$, may receive required amounts of resource res not only from an FDS located at a point $f d s$, as defined by a respective UR from a set (25), but also from an FDS located at a place $f d s^{\prime}$. As in all considered above cases, there may be $m \geq 1$ such alternative FDSs capable to deliver resource res to this FDS, and such opportunity is possible regarding any FDS connected with several supplying it FDSs.

At last, any reserve FPP, producing fuel res used by power plants for EP generation, located at a point $f p p^{\prime}$, may be represented by an UR

$$
\begin{aligned}
& \text { (res: } \left.f p p^{\prime}\right) \rightarrow \\
& n^{\prime} \cdot\left(\boldsymbol{k W h}: p t u^{\prime}\right) \text {, } \\
& m_{1}^{\prime} \cdot\left(\operatorname{res}_{1}^{\prime}: f s_{1}^{\prime}\right), n_{1}^{\prime} \cdot\left(\boldsymbol{k W h}: p t u_{1}^{\prime}\right) \text {, } \\
& m_{t}^{\prime} \cdot\left(\operatorname{res}^{\prime}{ }_{t}: f s^{\prime}{ }^{\prime}\right), \bar{n}^{\prime}{ }^{\prime}{ }^{\prime} \cdot\left(\boldsymbol{k W h}: p t u^{\prime}{ }^{\prime}\right),
\end{aligned}
$$

where $f s^{\prime}{ }_{1}, \ldots, f s^{\prime}{ }_{t}$, are points, where fuel storages with PECs $r e s^{\prime}{ }_{1}, \ldots, r e s^{\prime}{ }_{t}$ are located, so namely regarding these places power terminal units would be installed, thus providing relocation of amounts of these PECs necessary to an FPP for production of one unit of fuel res at a location $f p p^{\prime}$. The aforementioned relocation would be possible if needed amounts of electric power, i.e. $n_{1}^{\prime}, \ldots, n^{\prime}$, kilowatt-hours, would be available at points $p t u^{\prime}{ }_{1}, \ldots, p t u^{\prime}{ }_{t}$, where respective PTUs are operating. In turn, to produce one unit of a fuel res a reserve FPP itself would consume $n^{\prime}$ kilowatt-hours from a power terminal unit located at a point $p t u^{\prime}$.

Similarly to power plants, any fuel producing plant may be reserved not only by inclusion to an FI some additional FPPs but also by implementation of alternative ways of fuel producing and associated with them PECs, by which an FPP must be supplied. In this case for any UR (27) a unitary rule

$$
\begin{aligned}
& \text { (res: fpp) } \rightarrow \\
& n^{\prime} \cdot\left(\boldsymbol{k W h}: p t u^{\prime}\right) \text {, } \\
& m^{\prime}{ }_{1} \cdot\left(\operatorname{res}^{\prime}{ }_{1}: f s^{\prime}{ }_{1}\right), n^{\prime}{ }_{1} \cdot\left(\boldsymbol{k W h}: p t u^{\prime}{ }_{1}\right) \text {, } \\
& \text {... , } \\
& m_{t}^{\prime} \cdot\left(\operatorname{res}^{\prime}{ }_{t}: f s^{\prime}{ }_{t}\right), n^{\prime}{ }_{t} \cdot\left(\boldsymbol{k W h}: p t u^{\prime}{ }^{\prime}{ }^{\prime}\right),
\end{aligned}
$$

with the same header (res: $f p p$ ) and alternative body, representing a respective alternative supply set, necessary for this way implementation, is joined to a set $R_{E}$.

As may be seen, this generalization makes possible application of the criteria (80)-(85) to a general case of EIs without any corrections. The main difference is that UMGs representing such EIs are alternating, and thus $\bar{V}_{S_{q}}$ is a multi-element set of terminal multisets, i.e. $\left|\bar{V}_{S_{q}}\right| \geq 1$.

However, in practice an EI operates by some subset of it's components, and this subset as a whole has an ordinary concentric tree-like structure whilst the rest components stay in a reserve until an impact, after which some or even all of reserve components may be joined (switched) to an affected EI. To implement this approach it is sufficient to represent a reserved EI as a ternary tuple (for short "quadraple") $E=<\{\varnothing\}, R_{E}, v_{E}, v_{E}>$, where $\boldsymbol{v}_{\boldsymbol{E}}$ is a reserve resource base, any part (submultiset) of which $\Delta \boldsymbol{v}_{\boldsymbol{E}} \subseteq \boldsymbol{v}_{\boldsymbol{E}}$ may be added to an RB reduced by an impact, transforming it from $v_{E}-\Delta v$ to $v_{E}-\Delta v+\Delta v_{E}$. Following (64)-(67), a multiset $\Delta v_{E}$ may be represented as a sum of three non-intersecting multisets similar to MSs $v_{E}^{r e s}, v_{E}^{p}, v_{E}^{x}$ : 


$$
\Delta v_{E}=\Delta v_{E}^{r e s}+\Delta v_{E}^{p}+\Delta v_{E}^{x}
$$

the first summand

$$
\Delta \boldsymbol{v}_{E}^{r e s}=\left\{\boldsymbol{\Delta} \boldsymbol{M}_{1} \cdot\left(\operatorname{res}_{\boldsymbol{i}_{1}}: p_{\boldsymbol{i}_{1}}\right), \ldots, \boldsymbol{\Delta} \boldsymbol{M}_{\boldsymbol{s}} \cdot\left(\operatorname{res}_{\boldsymbol{i}_{s}}: p_{\boldsymbol{i}_{s}}\right)\right\}
$$

representing amounts of resources (PECs and fuels) having place at EI reserve fuel storages, as well as amounts of EP accumulated by backup power systems (in this case res $_{i_{j}}$ is nothing but $\boldsymbol{k W h}$ ); the second

$$
\Delta \boldsymbol{v}_{E}^{p}=\left\{\boldsymbol{\Delta} \boldsymbol{N}_{1} \cdot\left[p_{\boldsymbol{j}_{1}}, p_{\boldsymbol{j}_{1}}^{\prime}\right], \ldots, \boldsymbol{\Delta} \boldsymbol{N}_{\boldsymbol{u}} \cdot\left[p_{\boldsymbol{j}_{\boldsymbol{u}}}, p_{\boldsymbol{j}_{\boldsymbol{u}}}^{\prime}\right]\right\}
$$

representing reserve throughput capabilities of an EI (PTLs and pipes kept out of operation until an impact), and the third

$$
\Delta v_{E}^{x}=\left\{\Delta L_{1} \cdot\left(+x_{k_{1}}\right), \ldots, \Delta L_{z} \cdot\left(+x_{\boldsymbol{k}_{\mathbf{z}}}\right)\right\}
$$

representing reserve operation resource of an EI (facilities kept ready to operate if necessary). Thus addition to the reduced RB an MS $\Delta \boldsymbol{v}_{E}^{\text {res }}$ provides join to an affected EI some amounts of fuel located at reserve FSs, an MS $\boldsymbol{\Delta} \boldsymbol{v}_{E}^{p}-$ switching to an EI transporting network some reserve links (PTLs and pipes), and, at last, an MS $\boldsymbol{\Delta} \boldsymbol{v}_{E}^{x}-$ join to an EI some reserve producing, generating and transmitting/transferring facilities. After this addition criteria (80) - (82) may be applied to an EI $E=<\{\varnothing\}, R_{E}, v_{E}-\Delta v+\Delta v_{E}>$ and an order $q$. If this EI is not vulnerable then there exists an opportunity to recover it after impact, and thus there arises naturally a task of computation "the best" of all possible multisets $\Delta \boldsymbol{v}_{E} \subseteq v_{E}$ providing recovery of the affected EI to a state sufficient for an order completion. Otherwise it is clear that available reserve is insufficient for EI recovery and order completion.

\section{Modelling rechargeable power storages and their application}

Until now, introducing the concept of a power storage, we have not determined nor verbally, nor strictly, how power is accumulated in any specific PS, as well as how the last is recharged after or during order completion (for example, in a case of a car accumulator it is recharged during or, more correct, by a car motion). Let us consider a case of rechargeable power storages and their multigrammatical modelling..

Namely, we shall associate with any order $q$, which objective is meeting a demand on predefined by an external consumer collections of resources located at predefined places, a so called internal order $q^{\prime}$, which objective is addition of a collection $q^{\prime}$ to a resource base, i.e. full or partial (or even redundant) replenishment of resources spent whilst order $q$ completion. The simplest way of an internal order interpretation is a replacement of an RB $v_{E}$, remained after external order $q$ completion, by $v_{E}+q^{\prime}$. However, such approach is not satisfactory, because it, in fact, applies a concept of an energy infrastructure as an open system - a collection $q^{\prime}$ is not a part of EI resource base and is applied from systems which are external regarding EI. The second reason for rejection of this approach is that a collection $q^{\prime}$ is not at all correlated with an order $q$; it would be assigned to by an EI control system in some arbitrary way (the most natural one is $q^{\prime} \in \bar{V}_{S_{q}}$, that means full replenishment of spent resources).

So it would be necessary to develop such techniques of representation of logic of internal order $q^{\prime}$ construction and completion which, from one side, would provide it's compliance with an order $q$, and, from another one, would provide replenishment of namely power storages, applied for an order $q$ completion, by consumption of any other resources having place in an EI resource base. Such an approach fully fits the reality, where PSs are recharged on a regular basis by consumption of other resources entering an EI RB. Thus, firstly, a 
presumption that an EI is a closed system would be satisfied, and, secondly, all power storages, applied whilst order $q$ completion, would be recharged by means of only internal capabilities of an energy infrastructure.

The proposed multigrammatical representation of this important feature of EIs and their fragments is as follows. Let $v \in \bar{V}_{S_{q}}$ is a collection of resources consumed for an order $q$ completion, so $v \subseteq v_{E}$. We shall define a submultiset $v^{\prime}$ of a multiset $v$ in such a way that multiobjects entering $v^{\prime}$ represent amounts of power delivered from PSs during an order $q$ completion (all such multiobjects, obviously, have a form $n \cdot(\boldsymbol{k W h}: x)$ ), so

$$
v^{\prime}=\{n \cdot(\boldsymbol{k W h}: x) \mid n \cdot(\boldsymbol{k W h}: x) \in v\}
$$

Namely these amounts of electric power would be replenished in power storages before the next order would income to an EI, so this multiset is nothing but a needed internal order, i.e., for the first glance,

$$
v^{\prime}=q^{\prime}
$$

however, the substantial difficulty, breaking (101), is that to be an order, completed by some chain of energy transfers and transmissions, an MS $v^{\prime}$ in a general case would contain non-terminal objects, i.e. objects, being headers of unitary rules, representing an EI. At the same time an MS $v^{\prime}$, being a submultiset of an MS $v_{E}$, contains only terminal objects. To avoid this deadlock, we propose the following solution. To define logic of PSs replenishment (recharge) a set $R_{E}$ would contain URs of a form

$$
(* \boldsymbol{k W h}: x) \rightarrow X,
$$

where bold symbol "* " means that to replenish one kilowatt-hour at PS, located at a place $x$, it is sufficient to complete an order being a set, containing all multiobjects, entering a body $X$. So all URs like (102), in fact, define logic of PSs replenishment. If so, then, evidently,

$$
q^{\prime}=\left\{n \cdot(* \boldsymbol{k W h}: x) \mid n \cdot(\boldsymbol{k W h}: x) \in v^{\prime}\right\},
$$

so after an initial order $q$ completion, which results in delivery of determined by $q$ amounts of electric power, an internal order $q^{\prime}$ is completed, resulting in replenishment of power storages, applied during $q$ completion. As may be seen, power storages, applied during an internal order $q^{\prime}$ completion, are not replenished; otherwise a process of replenishment may become recursive and too complicated in an implementation. From the practical point of view this is quite natural. Let us note, as a conclusion of this Section, that not all power storages entering a considered energy infrastructure are rechargeable (i.e. in a multigrammatical representation of an EI not all URs with headers $(\boldsymbol{k} \boldsymbol{W h}: x)$ are supplemented by URs (102) with headers $(* \boldsymbol{k} \boldsymbol{W h}: x)$ ); all the rest PSs are presumed of a single use, so they may be replaced after consumption of all initially accumulated power.

\section{Conclusion}

As it was mentioned above, a criterial base, introduced in the Section 6, provides an assessment of vulnerability of energy infrastructures to destructive impacts: if an EI and an impact satisfy formulated conditions, then an EI is vulnerable; but if the aforementioned conditions are not satisfied, then it does not mean that an affected EI is substantially resilient to an impact. Let us underline, that we do not confirm, that in the case $\bar{V}_{S_{q}} \neq\{\varnothing\}$ an EI $E$ is capable to complete an order $q$, because in a general case there would be also assessed time delays associated with production and delivery of materiel resources (though regarding ElcI and electric power, circulating via it's networks and grids, in a general case such delays may be ignored). So, if an order includes a deadline for delivery of all necessary resources to external consumers being a source of this order, then, despite amounts of resources available for an order completion may be sufficient for this objective, even an optimal schedule of an 
order completion may not provide timely delivery of all necessary resources to consumers. This is an implication of non-additivity of time; time is an additive resource regarding separate device (facility), whilst regarding an EI as a whole it is non-additive because different devices may operate in parallel. So an EI, not satisfying the introduced above criteria and thus being not vulnerable in the above sense, in a general case may be not resilient to an impact. By this reason, if in a general case an order includes restrictions on duration of it's completion, then to assess EI resilience it would be necessary to apply more general mathematical tools than unitary multiset grammars. Such tools named temporal multiset grammars were for the first time announced in [3], and their application to the assessment of EIs resilience to destructive impacts will be considered in the future publications.

There is also an inverse task to be solved - namely, given a remained part of a considered EI segment and resources, to assess, what maximal subset of a full set of consumers may be provided by power and fuel in accordance with their demand. Another variation of this task is to assess whether some predefined part of consumers may be provided by power and fuel according to their demand while all the rest consumers may be provided by some part of their demand not less than some threshold values.

As well, in the future publications the aforementioned in the Section 7 task of optimal recovery of an affected EI will be considered, and also a task of an assessment of "the best" part of an order which may be completed given remained after an impact part of an EI (both with and without reserve).

The next extremely important task to be considered in future is a priori design of EIs being maximally resilient to the most expected sets (sequences) of impacts and consuming for a recovery minimally possible amounts of resources.

Let us note, that just the same techniques as described above in this paper regarding energy infrastructures may be applied also to heating systems, heating and cooling systems, combined heat and power systems $[35,36]$ and water supply systems [37-39]. An application of the MGF to these systems as well as to a sewer-mining [40] is described in short in [4]. All such partial applications would be joined in the near future to the integrated application of the multigrammatical framework to the area of resilience and recovery of critical infrastructures.

\section{Acknowledgements}

Author is grateful to Acad. Igor Bychkov, Acad. Yuriy Shokin, and Prof. Fred Roberts for useful discussions and support.

\section{References}

[1] Sheremet I A 2010 Recursive Multisets and their Applications (Moscow, Nauka) p 292 (In Russian)

[2] Sheremet I A 2011 Recursive Multisets and their Applications (Berlin, NG Verlag) p 249

[3] Sheremet I A 2018 Multiset Analysis of Consequences of Natural Disasters Impacts on Large-Scale Industrial Systems Data Science Journal 17(4) 1-17

[4] Sheremet I 2019 Multiset-based assessment of resilience of sociotechnological systems to natural hazards Natural Hazards Risks, Exposure, Response, and Resilience (London: IntechOpen). Available at: https://www.intechopen.com/books/natural-hazards-risk-exposure-response-and-resilience/multiset-basedassessment-of-resilience-of-sociotechnological-systems-to-natural-hazards (accessed: 10.06.2021)

[5] Sheremet I 2019 Multiset-Based Knowledge Representation for the Assessment and Optimization of LargeScale Sociotechnical Systems Enhanced Expert Systems (London: Intech Open) Available at: https://www.intechopen.com/books/enhanced-expert-systems/multiset-based-knowledge-representation-forthe-assessment-and-optimization-of-large-scale-sociotech (accessed: 10.06.2021)

[6] Sheremet I 2019 Unitary Multiset Grammars and Metagrammars Algorithmics and Applications Enhanced Expert Systems (London: Intech Open). Available at: https://www.intechopen.com/books/enhanced-expertsystems/unitary-multiset-grammars-an-metagrammars-algorithmics-and-application

[7] Sheremet I A 2021 Application of the Multigrammatical Framework to the Assessment of Resilience and Recoverability of Large-Scale Industrial Systems Resilience in the Digital Age. Lecture Notes in Computer Science (Springer) $\mathbf{1 2 6 6 0}$ pp 16-34 
[8] Wallace M 2002 Constraint logic programming Computational Logic: Logic Programming and Beyond. Lecture Notes in Computer Science (New York: Springer) 2407 pp 512-556

[9] Apt K 2003 Principles of Constraint Programming (Cambridge: Cambridge University Press) p 420

[10] Frunkwirth T, Abdennadher S 2003 Essentials of Constraint Programming (Berlin: Springer Verlag) p 398

[11] Kendal S L and Green M 2007 An Introduction to Knowledge Engineering (London: Springer) p 300

[12] Akerkar R and Sajja P 2010 Knowledge-Based Systems (Sudbury: Jones and Bartlett Publishers) p 350

[13] Bratko I 2012 Prolog Programming for Artificial Intelligence (New York: Addison Wesley) p 696

[14] Karlot J K 2005 Integer Programming: Theory and Practice (CRC Press) p 336

[15] Roberts F S 2009 Applied Combinatorics (London: Chapman \& Hall / CRC) p 860

[16] Lasdon S L 2013 Optimization Theory for Large Systems (New York: Dover Publications) p 560

[17] Taha H A 2016 Operations Research: An Introduction (London: Pearson) p 838

[18] Sheremet I 2020 Resource-based games Proceeding of the 2nd International Workshop on Information, Computation, and Control Systems for Distributed Environments (Irkutsk, Russia) 2638 pp 234-251

[19] Sheremet I 2020 Multigrammatical Approach to the Assessment of Sustainability of Intelligent Transport Systems 2020 Proceeding of the International Conference on Information Technology and Nanotechnology (Samara, Russia) pp 1-6

[20]Rinaldi S, Peerenboom J P and Kelly T K 2001 Identifying, understanding, and analyzing critical infrastructure interdependencies IEEE Control Systems Magazine 21 pp 11-25

[21] Katay ME 2010 Electric power industry as critical infrastructure Network World. Available at: https://www.networkworld.com/article/2217677/datacenter/electric-power-industry-as-criticalinfrastructure.html

[22] Nepal R and Jamasb T 2013 Security of European electricity systems: conceptualizing the assessment criteria and core indicators International Journal of Critical Infrastructure Protection 6(3-4) pp 182-196

[23] Larsen sen E R, Osorio S and van Ackere A 2017 A framework to evaluate security of supply in the electricity sector Renewable and Sustainable Energy Reviews 79 646-655

[24] He X and Cha E J 2018 Modeling the damage and recovery of interdependent critical infrastructure systems from natural hazards Reliability Engineering and System Safety 177 pp 162-175

[25] Liu K, Wang M, Zhu W, Wu J and Yan X 2018 Vulnerability analysis of an urban gas pipeline network considering pipeline road dependency International Journal of Critical Infrastructure Protection 22 pp 125138

[26] Rechak D, Senovsky P, Hromada M and Lovecek T 2019 Complex approach to assessing resilience of critical infrastructure elements International Journal of Critical Infrastructure Protection 25 pp 125-138

[27] Stergiopoulos G, Kotzanikolaou P, Theocharidou M, Lykou G and Gritzalis D 2016 Time-based critical infrastructure dependency analysis for large-scale and cross-sectoral failures International Journal of Critical Infrastructure Protection 12 pp 46-60

[28] Chomsky N 2005 Syntactic Structures (The Hague: Mouton de Gruyter) p 159

[29]Hopcroft J E, Motwani R and Ullman J 2001 Introduction to Automata Theory, Languages, and Computation (Reading, Mass.: Addison-Wesley) p 528

[30] Jesse B-J, Heinrichs H U and Kuckshinrichs 2019 Adapting the theory of resilience to energy systems: a review and outlook Energy, Sustainability and Society 9(27) pp 1-19

[31] Wang J, Zuo W, Rhode-Barbagidos L, Lu X, Wang J and Lin Y 2019 Literature Review on Modeling and Simulation of Energy Infrastructures from a Resilience Perspective Reliability Engineering and System Safety 183 pp 360-373

[32] Ji C, Wei Y and Poor H V 2017 Resilience of Energy Infrastructure and Services: Modeling, Data Analytics, and Metrics Proceedings of the IEEE 105(7) pp 1354-1366

[33] Brown R E 2008 Electric Power Distribution Reliability (Boca Raton, FL: CRC Press) p 110

[34] Lavaei J, Tse D and Zhang B 2014 Geometry of power flows and optimization in distributed networks IEEE Transactions on Power Systems 29(2) pp 572-583

[35] Werner S 2017 International review of district heating and cooling Energy 137 pp 617-631 
[36] Mazher A R, Liu S and Shukla A 2018 A state of art review on the district heating systems Renewable and Sustainable Energy Reviews 96 pp 420-439

[37] Kurian M and McCarney P 2010 Peri-urban Water and Sanitation Services. Policy, Planning and Method (New York: Springer) p 300

[38] Water supply. Available at: www.who.int.read/em2002chap7 (accessed: 10.06.2021)

[39] Water supply system. Encyclopedia Britannica. Available at: https://www.britannica.com/technology/watersupply-system (accessed: 10.06.2021)

[40] Makropoulos C, Rozos E, Tsoukalas I, Plevri A, Karakatsanis L and Karagiannidis L 2018 Sewer-mining: A water reuse option supporting circular economy, public service provision and entrepreneurship Journal of Environmental Management 216 pp 285-298 